\title{
Qualitative Business Surveys: Signal or Noise?
}

\author{
Silvia Lui, James Mitchell and Martin Weale* \\ National Institute of Economic and Social Research
}

September 30, 2009

\begin{abstract}
This paper identifies the information content at the firm level of qualitative business survey data by examining the consistency between these data and the quantitative data provided by the same respondents to the UK's ONS in official surveys. Since the qualitative data are published ahead of the quantitative data the paper then assesses the ability of the qualitative data to predict (or nowcast) the firm-level quantitative data.
\end{abstract}

Keywords: Early indicators; Firm-level comparison; Information content; Matched dataset; Qualitative business survey data; Quantitative official survey data

*Address for correspondence: National Institute of Economic and Social Research, 2 Dean Trench Street, Smith Square, London, SW1P 3HE, U.K. Tel: +44 (0) 207654 1926. Fax: +44 (0) 207654 1900. E-Mail: s.lui@niesr.ac.uk; j.mitchell@niesr.ac.uk and m.weale@niesr.ac.uk. We thank the CBI and the ONS for their help in facilitating this project, with particular thanks to Lai Co (CBI), Rhys Davies (ONS), Robert Gilhooly (ONS), Felix Ritchie (ONS), Eric Scheffel (ONS), Richard Welpton (ONS) and Jonathan Wood (CBI). We also thank the members of the project board, including representatives of the Bank of England, CBI and ONS, convened to advise this project, for helpful comments. Thanks also to three anonymous referees, an associate editor and participants in presentations at the National Statistics Methodology Advisory Committee (NSMAC), the CBI, the Bank of England and the MMF (Birkbeck, 2008), RES (Surrey, 2009) and ISAE (Rome, 2009) conferences. We gratefully acknowledge financial support from the ESRC (Award Reference: RES-062-23-0239) and the Bank of England. This work contains statistical data from ONS which are Crown copyright and reproduced with the permission of the controller of HMSO and Queen's Printer for Scotland. The use of the ONS statistical data in this work does not imply the endorsement of the ONS in relation to the interpretation or analysis of the statistical data. This work uses research datasets which may not exactly reproduce National Statistics aggregates. For more details about the CBI's ITS, please go to http://www.cbi.org.uk/ndbs/content.nsf/802737AED3E3420580256706005390AE/2F172E85D0508CEA80256E20003E95C6. 


\section{Introduction}

The purpose of this paper is to assess, at the firm level, the relationship between the qualitative business survey data produced by the Confederation of British Industry (CBI) collected in its Industrial Trends Survey (ITS) and related quantitative data collected by the Office for National Statistics (ONS) in its Monthly Production Inquiry (MPI) and used for the construction of the Index of Industrial Production (IoP). In the ITS firms are asked a range of questions to which they provide categorical instead of quantitative answers; for example they are asked whether output has fallen, stayed the same or risen but not by how much it has changed. Similar qualitative surveys exist in many other countries; indeed the CBI survey is the basis for the UK data maintained in the European Commission's database of business and consumer surveys for the European Union.

Interest focuses on the CBI survey and similar surveys as output indicators because, although qualitative by nature, they are seen as more timely than ONS data. In recent years this interest has increased as a result of doubts about official output data (see Ashley et al. (2005)). However past studies of the relationship with official output data have largely relied on comparisons between summary statistics from the qualitative survey (such as the extent to which the proportion of firms reporting a rise in activity exceeds the proportion reporting a decline, a figure normally computed after weighting the respondents by an indicator of their size and known as the balance statistic) and the percentage change in output reported by the ONS; see Driver \& Urga (2004) and, for a survey, Pesaran \& Weale (2006). At best, there have been comparisons between aggregate ONS data and the individual responses collected by the CBI but these too provide only a limited picture of the relationship between the two sets of data (see Mitchell et al. $(2002,2005,2006))$. Thus, on the basis of these studies it is difficult to say how firmly the perceived relationships between the two data sets are based.

In this paper, by contrast, we compare the individual responses provided to the CBI with those collected by the ONS on a firm-by-firm basis and test whether a relationship exists and, if so, identify its form. We explore the relationship between the two statistically. Then, to quantify the value to economists of any relationship between the two surveys we develop a means to assess the ability of the qualitative data to predict (or nowcast) the firm-level quantitative data. This represents an entirely new approach to provide a definitive answer to the question, how useful are qualitative surveys like the CBI's as indicators of output movements. Our focus is on the manufacturing sector since this is what the ITS covers, but, with suitable data a similar analysis could be carried 
out for other parts of the economy.

The plan of the remainder of the paper is as follows. Section 2 describes the matched panel dataset, discussing first the matching process and then examining the statistical properties of the matched dataset. This involves consideration of how representative the matched sample is of the economy as a whole. Section 2 concludes by describing when and how the data are transformed prior to analysis. Section 3 provides some simple descriptive evidence summarising the relationship between firms' qualitative and quantitative responses. Section 4 then supplements this with more formal econometric modelling which lets us both test whether the qualitative survey are noise, and helps us identify where the signal is strongest. Importantly, the models let us examine the dynamic relationship between the qualitative and quantitative data. A firm-level indicator of output growth used to evaluate the predictive power of the qualitative survey data is then introduced. Section 5 presents the econometric results and Section 6 concludes.

\section{The Matched Firm-level MPI and ITS Dataset}

\subsection{Background}

To match firms' responses across the two surveys we arranged for the CBI to provide the data collected by the ITS to the ONS in a manner which preserved obligations of confidentiality for both bodies. The ITS is a voluntary survey open to both CBI members and non-member companies. The ITS asks firms many questions, only some of which, like those on output, are 'verifiable' (i.e., testable against official data). Other questions in the ITS (e.g., "uncertainty about demand") cannot be verified. Nevertheless, we might deem it encouraging if we found that there were a strong signal from those questions which could be verified against official (ONS) data.

In this paper we focus on the question from the ITS which currently takes the form, "Excluding seasonal variations, what has been the trend over the past three months with regard to volume of output?". Firms reply "up", "same" or "down" (and "not applicable"). Firms are also asked to indicate how many employees they have within four size-bands: 0-199; 200-499; 500-4999 and 5000+ employees and we use this to provide extra information on our data. From the start of the survey in 1958 until June 2003 firms were asked about the trend in output over the last four months. From July 2003 onwards this was changed to three months as indicated above.

The CBI explained to its respondents that they would remain anonymous to users 
of the data and gave them the chance to opt out of having their responses matched to the ONS data. The data set available to us began in 2000 because the CBI changed its coding system at the start of 2000 and it was not possible to link data collected in 1999 and earlier with those collected from 2000 onwards. The CBI advised that respondents' co-operation was more likely if the data passed to ONS were not very recent and on these grounds asked its respondents to agree to the provision of data for the period 2000-2004. Only five respondents out of 2589 declined.

The ONS matched the CBI data set to its own MPI for the five years 2000-2004, inclusive. The MPI is a sample-based survey which asks close to 9000 firms each month for quantitative information on their turnover values in the month. Firms are also asked to indicate how many employees they have. The MPI uses stratified random sampling, stratifying the population by industry and employment. The MPI questionnaires are sent out to firms three days before the end of a calendar month; the majority of firms (the MPI achieves over a $80 \%$ response rate at the time of publication) then reply within 18 working days into the following month. In the event of non-response by firms which have responded previously, the ONS imputes a current value based on the average movement of other firms within the same industry, mean imputation rather than nearest neighbour. Over the five years of our sample period, pooled across firms about $11 \%$ of responses to the turnover question in the MPI sample are imputed.

The MPI asks firms about their turnover while the ITS asks about output. The difference between these is accounted for by the change in stocks of finished goods and work in progress. The ONS measures this by means of a separate Quarterly Inventories Inquiry and uses a small monthly inquiry to supplement these data so as to produce the monthly figures needed to deliver the IoP (ONS 2007). The adjustments are applied at an industry level and not at a firm level, however. This means that the comparison that we make is between monthly sales as reported to the ONS and the response to the ITS which should indicate what is happening to output. When fluctuations in sales growth are not met from stocks, and lead directly to output movements, the MPI and the ITS responses should have a close direct relationship. But, as we explain in Section 4, we should still expect the MPI and ITS responses to be related despite the effects of stock changes. We provide further information on the two data sets in appendices A.1 and A.2.

No information is available on precisely who, at a given firm, fills out the survey form nor on how often this person changes over time. The CBI survey is generally replied to by a board member, while the ONS survey, at least for larger firms, may be filled in at a lower level. 
The IoP is published on the 26th working day after the end of the reference month with the consequence that it is published more than a calendar month after the month to which it relates; see ONS (2005) for details. The ITS is published at the end of the month concerned, and it therefore gives an impression of being more than a calendar month ahead of the MPI. This explains the potential value of the ITS as a timely source of information about the current state of the economy.

However, firms fill in their ITS forms between the beginning of the last week of the preceding month and the middle of the current month. As a result the ITS does not cover all of the month in which it is published. Coupled with a longer reference period (as firms in the ITS are asked about the last three/four months) it becomes apparent that while the ITS is published ahead of the MPI it need not contain more timely information about economic activity in the current month. The desire to examine this question motivates the empirical work below that tests, for the first time, the informational content of these ITS firm-level data.

\subsection{The matching process}

The matched ITS/MPI dataset, the focus of our statistical and econometric analysis, was constructed by first matching the panel of firms which replied to the ITS against the ONS's Interdepartmental Business Register (IDBR). The IDBR is a list of UK businesses accounting for almost $99 \%$ coverage of economic activity (ONS 2009). It covers all parts of the economy, but excludes some of the very small businesses. They are usually those of the self-employed, those without employees and with low turnover. Some non-profit making organisations are also excluded. In Table 1 we provide statistics on the number of responding firms in each year in the MPI, the ITS, the ITS firms matched to the IDBR and the subset of these which matches the MPI data set. These are provided for the start of each year and also at the end of our data set.

Column A summarises the responses to the MPI at the start of each year and also shows the total number of distinct respondents over the five-year period. Column B in Table 1 provides similar data for the ITS. As well as ignoring the five firms who opted out, and whose response pattern we do not know, these figures also omit responses to twenty-five ID numbers in the ITS data set used for anonymous responses; these IDs may represent responses from more than one firm.

Text matching based on common variables, specifically the names, addresses and postcodes of firms, was used to match these CBI firms against those firms on the ONS's IDBR. 
Table 1: The number of firms in the raw datasets and the matched response dataset

\begin{tabular}{|c|c|c|c|c|}
\hline Year & $\begin{array}{c}\text { A } \\
\text { MPI firms }\end{array}$ & $\begin{array}{c}\text { B } \\
\text { ITS Firms }\end{array}$ & $\begin{array}{c}\text { C } \\
\text { ITS Firms uniquely } \\
\text { Matched to IDBR }\end{array}$ & $\begin{array}{c}\text { D } \\
\text { Matched ITS } \\
\text {-MPI Firms }\end{array}$ \\
\hline Jan 2000 & 9005 & 614 & 445 & 152 \\
\hline Jan 2001 & 9046 & 783 & 575 & 163 \\
\hline Jan 2002 & 8959 & 841 & 623 & 198 \\
\hline Jan 2003 & 8916 & 820 & 608 & 158 \\
\hline Jan 2004 & 8913 & 796 & 588 & 176 \\
\hline Dec 2004 & 8641 & 750 & 585 & 160 \\
\hline $\begin{array}{l}\text { Total No. } \\
\text { Distinct } \\
\text { Respondents }\end{array}$ & 28033 & 2584 & 1895 & 807 \\
\hline $\begin{array}{l}\text { Turnover } \\
\text { Rate (\% p.a.) }\end{array}$ & $44 \%$ & $48 \%$ & $47 \%$ & $77 \%$ \\
\hline
\end{tabular}

Of the 2584 different firms who replied at least once to the ITS 2120 (82\%) were initially matched against the IDBR; we are, however, not able to allocate these across individual months. Of these 2120 firms, as column C indicates, there was a 'definite' match (defined as when the ONS is at least $80 \%$ confident in the match) between the ITS firms and the IDBR for 1895 firms, i.e., for about $90 \%$ of firms. For the remaining 464 firms, there was a non-unique mapping between their ITS and IDBR reference numbers. This obviously creates confusion about whether a given firm in the IDBR is the same firm as in the ITS; on the advice of the ONS these firms were therefore dropped from our analysis to minimise the risk of matching errors. The ONS quantify their confidence in a match based on the percentage of these common variables (with the name and address of a firm, which themselves can be compounds, assigned a higher weight than the postcode) which are matched successfully across the two surveys using matching software produced by Search Software America.

The subset of ITS firms uniquely matched to the IDBR which are also sampled by the MPI could then be extracted in the second stage of the matching. 807 different firms gave at least one contemporaneous matched response to the ITS and MPI. Column D in Table 1 shows that, on average across years, there are about 170 different firms present in the matched dataset each month. Over the five years, as 807 different firms make the matched ITS-MPI dataset and 2584 different firms were sampled at least once by the 
CBI, the match rate against the ITS is $31 \%$ although the average monthly match rate was $22 \%$. There is a total of 10254 responses provided by these 807 firms. 430 of these or about $4 \%$ are imputations provided by ONS. We have not distinguished these from the hard data in the dataset.

The turnover rates shown in the last row of Table 1, and discussed further below, are calculated as follows. Consider first the case where $Y_{m, 1}$ indicates the number of firms in the dataset of interest (column A-D) responding in the first month of year $m$ and we have data for $M$ years $(m=1, \ldots, M)$. $\tilde{Y}$ is the total number of firms responding in all $M$ years of the survey. We can then define the turnover rate as the value of $r$ which satisfies

$$
\sum_{m=1}^{M} Y_{m, 1}-(1-r) \sum_{m=1}^{M-1} Y_{m, 1}=\tilde{Y}
$$

However, in our case our last observation is for the last month of year $M-1$ rather than the first month of year $M$; we denote this $Y_{M-1,12}$. We therefore define the turnover rate, $r$, as that value which satisfies

$$
\sum_{m=1}^{M-1} Y_{m, 1}+Y_{M-1,12}-(1-r) \sum_{m=1}^{M-2} Y_{m, 1}-(1-r)^{\frac{11}{12}} Y_{M-1,1}=\tilde{Y}
$$

reflecting the fact that the interval between $Y_{M-1,1}$ and $Y_{M-1,12}$ is only eleven months.

\subsection{Statistical properties of the matched ITS-MPI dataset}

We summarise the characteristics of the matched dataset with a number of statistics. First we explore how many respondents in the matched data set provide responses in more than one period; since we are interested in an econometric analysis involving lag terms we are particularly interested in knowing how many respondents provide runs of consecutive responses. And secondly we discuss the extent to which the matched sample is representative of the economy as a whole.

The total number of times a given firm, firm $i(i=1, \ldots, N)$, is present in the matched dataset, $T_{i}$, ranges from 1 to 60 . The average number (across firms) of matched timeseries observations is 12.7 , i.e. $\overline{T_{i}}=12.7$. $25 \%$ of the 807 firms have at most 3 matched (contemporaneous) responses; $50 \%$ have at most 8 matched responses and $75 \%$ have at most 16 matched responses. This response pattern is summarised in Figure 1.

However, consecutive responses are much less frequent. In the matched dataset 96 


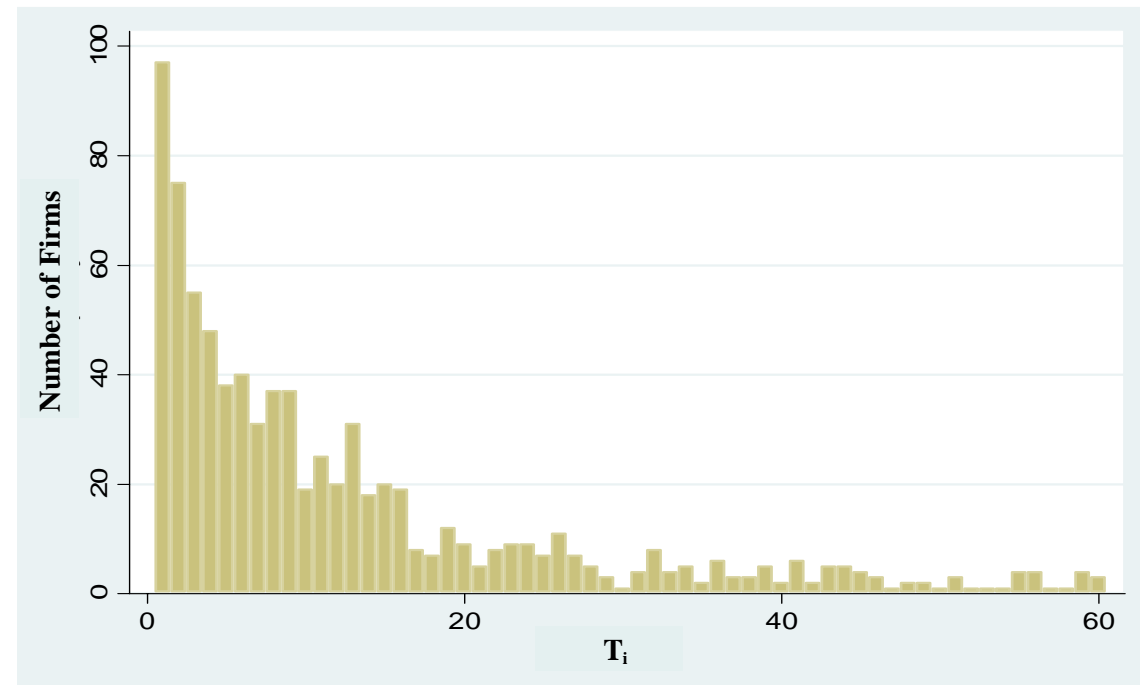

Figure 1: Histogram indicating the number of firms with $T_{i}$ responses in the matched dataset

firms reply to at least twelve consecutive observations (or thirteen in the level of turnover). These 96 firms have 1033 observations between them (i.e., pooled across time).

A feature of Table 1 is that the turnover rate in the matched sample is much higher than in either the ITS or MPI. However, if we focus on the retention rate, as one minus the turnover rate, we note that the retention rate for the matched data set is at $23 \%$ not much lower than the product of the retention rates of the MPI and the ITS, which is $29 \%$. The latter is the retention rate which would be expected from large samples if turnover were driven by independent processes in the two surveys. The lower actual retention rate implies that a firm is more likely to remain in the ITS sample if it drops out of the MPI sample than if it remains in and vice versa; we have been unable to identify reasons for this and note that, to the extent that turnover is driven by a common cause such as survival discussed below, the retention rate in the matched data set would be expected to be higher than that calculated on the assumption of independence.

Table 2 indicates the average (across the years 2000-2004, inclusive) size of firms in the matched ITS-MPI dataset, including the sub-panel of 96 firms with at least 12 consecutive 
Table 2: Proportion of firms in different employment size bands (averaged across years 2000-2004 inclusive)

\begin{tabular}{lllll}
\hline Band & 1 & 2 & 3 & 4 \\
Employment Size & 0 to 199 & 200 to 499 & 500 to 4999 & $\geq 5000$ \\
\hline MPI & $75.40 \%$ & $16.6 \%$ & $7.8 \%$ & $0.2 \%$ \\
ITS & $76.50 \%$ & $14.8 \%$ & $7.8 \%$ & $0.9 \%$ \\
Matched Firms & $53.57 \%$ & $32.2 \%$ & $13.6 \%$ & $0.7 \%$ \\
96-firm Sub-panel of Matched Firms & $42.52 \%$ & $45.0 \%$ & $12.0 \%$ & $0.5 \%$ \\
\hline
\end{tabular}

responses. Table 2 also reports the average size of firms in the ITS and MPI. Since the size of a firm can change over time, in a given year we take an average of the available monthly observations. For the MPI these observations are quantitative and we take the mean, while for the ITS they are categorical and we take the mode. Taking the modal value from the MPI led to similar results.

Table 2 shows that the MPI and ITS have similar characteristics, although the ITS does sample more large firms. But the matched ITS-MPI dataset contains a far bigger proportion of large firms than the MPI. Around three-quarters of firms in the MPI have fewer than 200 employees, as opposed to around one half in the matched dataset. The proportion of firms with fewer than 200 employees is even lower in the 96 firm sub-panel. The matched dataset is therefore not representative of UK manufacturing, as characterised by the MPI, since it over-represents large firms.

This over-representation of large firms in the matched dataset is explained by a number of factors. First, the stratified sample design of the MPI ensures that larger firms are more likely to remain in the sample and thus contribute to both the matched dataset and the subset of matched firms with at least 12 consecutive monthly responses. Secondly, it is easier to match the larger (well-known) firms than the smaller firms. Recall the ONS classify a match as "definite" when their matching algorithms have $80 \%$ confidence in the match. For those firms that make the matched dataset, $13 \%$ of firms with fewer than 200 employees have a confidence score less than $95 \%$; whereas only $8 \%$ of firms with more than 500 employees have a score less than $95 \%$.

In Table 3 we shed further light on why the bulk of firms (807-96=711 firms) do not survive the twelve-month period, leading to the high turnover rate for the matched dataset seen in Table 1. This table is constructed from the ONS's Business Structure Database (BSD). The BSD contains successive (yearly) vintages of the IDBR, and provides an historical record of the life-span and structure of firms.

Table 3 shows the percentage of these 711 firms that died or experienced some form 
Table 3: The percentage of matched firms, which do not make the sub-panel of 96 firms with at least 12 consecutive responses, that died or experienced demographic change in the year following their presence in the matched dataset

\begin{tabular}{lcrrrr}
\hline Year present in matched sample & 2000 & 2001 & 2002 & 2003 & 2004 \\
Year present in the BSD & 2001 & 2002 & 2003 & 2004 & 2005 \\
& & & & & \\
$\begin{array}{l}\text { Percentage of firms } \\
\text { that died or experienced demographic change }\end{array}$ & $17.76 \%$ & $21.29 \%$ & $15.99 \%$ & $13.68 \%$ & $13.80 \%$ \\
\hline
\end{tabular}

of demographic change (such as a takeover, merger or re-structuring including a change in ownership) in the year following their presence in the matched dataset. We find that across the five years, $14 \%-21 \%$ of these firms drop out of the matched dataset because of death or demographic change. This implies that the high turnover rate observed for the matched dataset is explained, in large part, not by death or demographic change, but living firms not replying to the MPI and ITS simultaneously.

In summary, this evidence suggests that the matched ITS-MPI dataset, with its strong bias towards large firms, is not a random sample from the population of UK manufacturing firms, represented by the MPI. But this does not mean that the matched dataset picked up firms that were either particularly good or bad at replying to the ITS. What matters is whether the relationship between the ITS and MPI data, at a firm-level, depends on the probability that a firm is in the matched dataset; in other words, whether the relationship depends on the type of firm under consideration. It is therefore important in the econometric analysis below to undertake econometric tests for sample selection; an issue which we explore in Section 5.1.4. Beyond this, the bias towards large firms is not in itself a problem since a finding that the ITS and the MPI responses were not related in our matched sample would be of considerable interest even if it applied only to large firms and nothing were known about small firms.

\subsection{Further statistical issues}

Before comparison of the two surveys can be carried out using these matched data a range of statistical issues needs to be addressed. The firm-level MPI turnover data are measured in current prices and have to be converted to constant prices by means of appropriate output price indices. The ONS classifies each MPI respondent to a particular industry 
at a 4-digit level in the Standard Industrial Classification (the most detailed level of the hierarchical classification). This classification is based on the nature of the firm's principal product and the ONS has provided an output price index for each 4-digit SIC category with the price indices selected on the basis of each firm's current industrial classification. We have used these price indices to convert the turnover data to measures at constant prices.

The firm-level MPI data are not seasonally adjusted while respondents to the ITS report "after taking seasonal effects into account". In the absence of seasonally adjusted data from the ONS at the firm level, we prefer to address seasonality by using appropriate dummy variables in the models we use to assess the relationship between the MPI and ITS data (except in the case where we consider differences over 12 months since in this case the seasonal effects are removed). Specifically, let $x_{i, t}$ denote the volume of turnover for firm $i$ for time (month) $t(t=1, \ldots, T$, where $T=60$ months in our application), computed from the MPI. The $k$-month growth rate is defined as

$$
z_{i, t}^{(k)}=\ln x_{i, t}-\ln x_{i, t-k}, \quad(k=1, \ldots, 12),
$$

while the rolling 1-month growth rate, which we also consider to facilitate interpretation, is defined as

$$
\Delta x_{i, t-k}=\ln x_{i, t-k}-\ln x_{i, t-k-1}=z_{i, t-k}^{(1)},(k=0, \ldots, 11) .
$$

In addition, 2.5\% Winsorisation on each of the upper and lower tails of the distribution of the turnover growth rates, pooled across firms for each period, is carried out prior to analysis to mitigate the possible effects of outliers. Two-tailed Winsorisation (Dixon 1960) involves replacing those values of a variable below the lower or above the higher $x$-percentile with the values observed at those percentiles. It is generally preferred to trimming as a means of dealing with outliers. We discuss the effects of Winsorisation as we present our results.

\section{Descriptive statistics}

Table 4 shows, pooled across firms and time with $\sum_{i=1}^{N} T_{i}$ denoting the size of the pooled sample, the average (quantitative) turnover growth rate in both the Winsorised and nonWinsorised MPI datasets, conditional on firms' qualitative answers to the ITS. Results are shown both for the full matched panel set and the sub-sample of 96 firms from the 
full sample with 12 consecutive responses which we examine further below. In both cases, we see that when relating the ITS to turnover growth over the last month, i.e. $\Delta x_{i, t}$, the firms who replied to the ITS saying their turnover had gone up did not experience turnover growth greater than that of other firms. For example, over the full matched non-Winsorised sample, firms reporting falls, in reality, saw their mean deflated turnover rise by $0.53 \%$ while firms reporting rises saw their mean turnover fall, but only slightly, by $-0.08 \%$.

However, when relating firms' qualitative answers to $\Delta x_{i, t-1}$ we do see that firms reporting rises experienced greater mean growth than firms reporting falls, although only for $\Delta x_{i, t-2}$ do we also see those firms who reported their output had not changed experience turnover growth in between the means for those reporting rises and those reporting falls. In addition, at $\Delta x_{i, t-2}$ we observe larger differences between the turnover growth rates of optimistic and pessimistic firms. The only differences between the average growth rates in the different categories which are statistically significant at a $95 \%$ level (with $t$-values $>1.96)$ are those for period $t-2$ between the mean growth rate for those firms reporting down and up, or the same and up, from the full sample, whether Winsorised or not and from the sub-panel of 96 firms after Winsorisation. These calculations are made using the variance of the difference between the means computed as the sums of the variances of the means for the two categories compared. The variance estimates can be added due to independence (the sample means for each categorical response are based on disjoint sets of observations).

\section{Assessment of the Reliability of the ITS Data}

To assess formally the reliability of the CBI data we model the relationship between the ITS and MPI data, allowing for their dynamics, and test various hypotheses about the former. The analysis takes no specific account of the sampling design of either the MPI or the ITS.

The ITS asks each firm, $i\left(i=1, \cdots, N_{t}\right)$ at time $t(t=1, \cdots, T)$, to give qualitative answers to the question about its trend of output (excluding seasonal variations) over the past three/four months. As discussed, the firm can respond either 'up', 'same' or 'down', denoted as $j$ (where $j=2,1,0$, respectively). Firms can, and a very small number do, also respond 'not applicable'. We ignore these firms below.

We then assume that there is a continuous latent variable $y_{i, t}^{*}$ that triggers firm $i$ 's 
Table 4: Mean growth rate $(\times 100)$ for MPI turnover given firms' qualitative responses to the ITS

\begin{tabular}{|c|c|c|c|c|c|c|c|c|c|}
\hline \multicolumn{10}{|c|}{ Prior to Winsorisation (full matched panel) } \\
\hline & $\begin{array}{l}\Delta x_{i t} \\
\text { down }\end{array}$ & same & up & $\begin{array}{c}\Delta x_{i t-1} \\
\text { down }\end{array}$ & same & up & $\begin{array}{c}\Delta x_{i t-2} \\
\text { down }\end{array}$ & same & up \\
\hline Mean & 0.53 & 0.65 & -0.08 & 0.27 & 0.19 & 0.72 & -1.71 & 0.19 & 3.11 \\
\hline SD of Mean & 0.86 & 0.67 & 0.76 & 0.97 & 0.79 & 0.86 & 1.00 & 0.79 & 0.90 \\
\hline$\sum_{i=1}^{N} T_{i}$ & 1977 & 2948 & 1862 & 1464 & 2246 & 1389 & 1388 & 2160 & 1352 \\
\hline \multicolumn{10}{|c|}{$2 \frac{1}{2} \%$ upper and lower tails Winsorised (full matched panel) } \\
\hline & $\begin{array}{c}\Delta x_{i t} \\
\text { down }\end{array}$ & same & up & $\begin{array}{c}\Delta x_{i t-1} \\
\text { down }\end{array}$ & same & up & $\begin{array}{c}\Delta x_{i t-2} \\
\text { down }\end{array}$ & same & up \\
\hline Mean & 0.65 & 0.46 & 0.06 & -0.15 & 0.72 & 0.79 & -1.20 & 0.13 & 2.64 \\
\hline SD of Mean & 0.56 & 0.44 & 0.56 & 0.64 & 0.49 & 0.63 & 0.68 & 0.51 & 0.65 \\
\hline$\sum_{i=1}^{N} T_{i}$ & 1977 & 2948 & 1862 & 1464 & 2246 & 1389 & 1388 & 2160 & 1352 \\
\hline \multicolumn{10}{|c|}{ Prior to Winsorisation (sub-panel of 96 firms) } \\
\hline & $\begin{array}{c}\Delta x_{i t} \\
\text { down }\end{array}$ & same & up & $\begin{array}{c}\Delta x_{i t-1} \\
\text { down }\end{array}$ & same & up & $\begin{array}{c}\Delta x_{i t-2} \\
\text { down }\end{array}$ & same & up \\
\hline Mean & 2.33 & -0.29 & -0.06 & -0.61 & -0.01 & 0.89 & -4.08 & -0.08 & 4.12 \\
\hline SD of Mean & 3.87 & 2.39 & 2.51 & 3.66 & 2.49 & 2.51 & 3.63 & 2.42 & 2.52 \\
\hline$\sum_{i=1}^{N} T_{i}$ & 264 & 469 & 300 & 264 & 469 & 300 & 264 & 469 & 300 \\
\hline \multicolumn{10}{|c|}{$2 \frac{1}{2} \%$ upper and lower tails Winsorised (sub-panel of 96 firms) } \\
\hline & $\begin{array}{c}\Delta x_{i t} \\
\text { down }\end{array}$ & same & up & $\begin{array}{c}\Delta x_{i t-1} \\
\text { down }\end{array}$ & same & up & $\begin{array}{c}\Delta x_{i t-2} \\
\text { down }\end{array}$ & same & up \\
\hline Mean & 1.23 & 0.22 & -0.41 & -1.88 & 1.25 & 0.38 & -2.04 & -1.15 & 3.14 \\
\hline SD of Mean & 1.62 & 1.11 & 1.44 & 1.60 & 1.16 & 1.46 & 1.55 & 1.15 & 1.44 \\
\hline$\sum_{i=1}^{N} T_{i}$ & 264 & 469 & 300 & 264 & 469 & 300 & 264 & 469 & 300 \\
\hline
\end{tabular}

Notes: SD of Mean denotes the standard deviation of the sample mean. $\sum_{i=1}^{N} T_{i}$ denotes the size of the sample pooled across firms and time. The 96 firm sub-panel consists of those firms in the matched dataset with at least twelve consecutive observations. 
categorical response at time $t$ via the following observation rule:

$$
y_{i, t}=j \text { if } \mu_{j}<y_{i, t}^{*} \leq \mu_{j+1}, j=0,1,2,
$$

where $\mu_{j}$ 's are the unknown thresholds to be estimated: $\mu_{0}=-\infty, \mu_{j} \leq \mu_{j+1}$ and $\mu_{3}=\infty$.

The latent variable $y_{i, t}^{*}$, as is set out in our model in equation (6) or (7), is then assumed to depend on both a firm's contemporaneous and lagged turnover growth, as measured by the MPI, and, to account for inertia, its previous qualitative replies to the ITS. Temporal dependence in $y_{i, t}^{*}$ is captured via the lagged dummy variables $y_{i, t}^{(j)}$ (one for $j=0$ and one for $j=2$, with $j=1$ excluded to avoid collinearity). These take a value 1 if $y_{i, t}=j$ and 0 otherwise. More general dynamics are picked up by considering lags of the explanatory variables. We start with lags of up to twelve months in $z_{i, t}$ and $\left(y_{i, t}^{(0)}, y_{i, t}^{(2)}\right)$ and assume that $y_{i, t}^{*}$ provides noisy estimates of the quantitative data, $z_{i, t}^{(k)}$; thus we consider the following general model:

$$
\begin{aligned}
y_{i, t}^{*}= & \beta_{1} z_{i, t}^{(1)}+\beta_{2} z_{i, t}^{(2)}+\cdots+\beta_{12} z_{i, t}^{(12)}+\lambda_{1}^{(0)} y_{i, t-1}^{(0)}+\ldots+\lambda_{12}^{(0)} y_{i, t-12}^{(0)} \\
& +\lambda_{1}^{(2)} y_{i, t-1}^{(2)}+\ldots+\lambda_{12}^{(2)} y_{i, t-12}^{(2)}+\alpha_{i}+\epsilon_{i, t}
\end{aligned}
$$

or, equivalently, expressed in terms of month-on-month growth rates:

$$
\begin{aligned}
y_{i, t}^{*}= & \gamma_{1} \Delta x_{i, t}+\gamma_{2} \Delta x_{i, t-1}+\cdots+\gamma_{12} \Delta x_{i, t-11}+\lambda_{1}^{(0)} y_{i, t-1}^{(0)}+\ldots+\lambda_{12}^{(0)} y_{i, t-12}^{(0)} \\
& +\lambda_{1}^{(2)} y_{i, t-1}^{(2)}+\ldots+\lambda_{12}^{(2)} y_{i, t-12}^{(2)}+\alpha_{i}+\epsilon_{i, t}
\end{aligned}
$$

where

$$
\gamma_{j}=\sum_{k=j}^{12} \beta_{k}
$$

and the model nests the special, and testable, case that the ITS data relate, as the CBI ask, to growth over the last three (previously four) months.

$\alpha_{i}$ is a firm-specific and time-invariant random component such that $\alpha_{i} \sim N\left(0, \sigma_{\alpha}^{2}\right)=$ $f\left(\alpha_{i}\right)$, which accommodates heterogeneity (across firms) in the thresholds $\mu_{1}$ and $\mu_{2} . \epsilon_{i, t}$ is a time and firm-specific error term which is assumed to be normally distributed and uncorrelated across firms and uncorrelated with $\alpha_{i}$. The variance of $\epsilon_{i, t}$ is set to unity for identification, so that $E\left(\epsilon_{i, t}, \epsilon_{i, s}\right)=\rho=\sigma_{\alpha}^{2} /\left(\sigma_{\alpha}^{2}+1\right)$ for $t \neq s$. We focus on equation (7), rather than (6), since interpretation is perhaps easier, as it breaks down the cumulative effect of turnover growth over the last $k$ month into the month-on-month impact in the last $k$ months and thereby helps us track down the source of the signal. Appendix A.3 
explains that this general model, (7) can also be motivated as the solution of a twoequation model which accommodates the potential endogeneity of sales/turnover growth, as long as a sufficient number of lagged terms in $\Delta x_{i, t}$ are included.

The assumption that $\epsilon_{i, t}$ is independent across firms, $i$, while commonly made in applied work, is restrictive. Independence rules out common shocks that affect all firms' responses. In the spirit of Pesaran (2006), who suggested augmenting the panel data model with cross-sectional averages of the dependent and independent variables, we seek to accommodate cross-sectional dependence by letting $\epsilon_{i, t}$ depend on the balance statistic, $B a l_{t}$, and aggregate output growth, $\Delta x_{t}$, (and in principle their lags), such that

$$
\epsilon_{i, t}=\delta_{1} B a l_{t}+\delta_{2} \Delta x_{t}+v_{i, t}
$$

where $v_{i, t}$, an idiosyncratic shock, is normally distributed white noise and uncorrelated across firms; $\mathrm{Bal}_{t}$ is defined as the proportion of firms reporting a rise in output less the proportion reporting a fall and $\Delta x_{t}$ is aggregate growth. Firms' sentiment as characterised by the ITS, in other words, may have a common collective component, as well as individual components; see also Lui et al. (2009).

The series for $\mathrm{Bal}_{t}$ is computed from the ITS and available, together with similar data for other European countries, from the DG-ECFIN website

$$
\text { (http://ec.europa.eu/economy_finance/db_indicators/surveys9185_en.htm); }
$$

firms' responses are weighted by sales data provided to the CBI and the aggregate is seasonally adjusted. The series for $\Delta x_{t}$ is the first difference of the logarithm of the manufacturing component of the IoP (series CKYY on the ONS database) and takes account of changes in stocks of finished goods.

To estimate (7), given (5) and the distributional assumption about $v_{i, t}$, we derive the probabilities from the conditional distribution of $y_{i, t}^{*}$ on $\Omega_{i, t}$, where $\Omega_{i, t}$ is the information available to firm $i$ up to and including time $t$ :

$$
\begin{aligned}
P\left(y_{i, t}=\right. & \left.j \mid \Omega_{i, t}\right)=P\left(\mu_{j}<y_{i, t}^{*} \leq \mu_{j+1} \mid \Omega_{i, t}\right)=P_{j i, t}= \\
= & \Phi\left(\begin{array}{c}
\mu_{j+1}-\gamma_{1} \Delta x_{i, t}-\cdots-\gamma_{12} \Delta x_{i, t-11}-\lambda_{1}^{(0)} y_{i, t-1}^{(0)}-\ldots-\lambda_{12}^{(0)} y_{i, t-12}^{(0)} \\
-\lambda_{1}^{(2)} y_{i, t-1}^{(2)}-\ldots-\lambda_{12}^{(2)} y_{i, t-12}^{(2)}-\delta_{1} B a l_{t}-\delta_{2} x_{t}-\alpha_{i}
\end{array}\right) \\
& -\Phi\left(\begin{array}{c}
\mu_{j}-\gamma_{1} \Delta x_{i, t}-\cdots-\gamma_{12} \Delta x_{i, t-11}-\lambda_{1}^{(0)} y_{i, t-1}^{(0)}-\ldots-\lambda_{12}^{(0)} y_{i, t-12}^{(0)} \\
-\lambda_{1}^{(2)} y_{i, t-1}^{(2)}-\ldots-\lambda_{12}^{(2)} y_{i, t-12}^{(2)}-\delta_{1} B a l_{t}-\delta_{2} x_{t}-\alpha_{i}
\end{array}\right),
\end{aligned}
$$


where $\Phi($.$) denotes the standard normal cumulative density function.$

The log-likelihood function, following Butler \& Moffit (1982), is then given as

$$
\ln L=\sum_{i=1}^{N} \ln \int_{-\infty}^{\infty} \prod_{t=1}^{T}\left(P_{0 i, t}^{y_{i, t}^{0}} P_{1 i, t}^{y_{i, t}^{1}} P_{2 i, t}^{y_{i, t}^{2}}\right) f\left(\alpha_{i}\right) d \alpha_{i}
$$

where $y_{i, t}^{j}=1$ if $\mu_{j}<y_{i, t}^{*} \leq \mu_{j+1}, 0$ otherwise, $j=0,1,2$ and $N$ is the total number of different firms present over time $(t=1, \ldots, T)$.

Under the above assumptions, maximisation of (11) yields consistent estimates $(N, T \rightarrow$ $\infty)$ for the coefficients denoted: $\widehat{\sigma}_{\alpha}^{2}, \hat{\mu}_{j}(j=0, \ldots, 3), \widehat{\gamma}_{l}(l=0, \ldots, 11), \widehat{\lambda}_{m}^{j}(m=1, \ldots, 12$, $j=0$ and 2$), \widehat{\delta}_{1}$ and $\widehat{\delta}_{2}$.

To validate assumptions implicit in (7) and (9) we carry out diagnostic tests on the generalised residuals from (7) and (9). The generalised residuals of Gourieroux et al. (1987) are given by

$$
\begin{aligned}
E\left(v_{i, t} \quad \mid \quad y_{i, t}=j, \Delta x_{i, t}, \ldots, \Delta x_{i, t-11,} y_{i, t-1}^{(0)}, \ldots, y_{i, t-12}^{(0)}, y_{i, t-1}^{(2)}, \ldots, y_{i, t-12}^{(2)}, B a l_{t}, x_{t}\right)= \\
=\frac{\widehat{\phi}\left(\mu_{j}-\Theta\right)-\widehat{\phi}\left(\mu_{j+1}-\Theta\right)}{\widehat{\Phi}\left(\mu_{j+1}-\Theta\right)-\widehat{\Phi}\left(\mu_{j}-\Theta\right)}
\end{aligned}
$$

where $\phi$ denotes the standard normal density function,

$$
\begin{aligned}
\Theta= & \gamma_{1} \Delta x_{i, t}+\gamma_{2} \Delta x_{i, t-1}+\cdots+\gamma_{12} \Delta x_{i, t-11}+\lambda_{1}^{(0)} y_{i, t-1}^{(0)}+\ldots+\lambda_{12}^{(0)} y_{i, t-12}^{(0)}+\lambda_{1}^{(2)} y_{i, t-1}^{(2)}(13) \\
& +\lambda_{12}^{(2)} y_{i, t-12}^{(2)}+\alpha_{i}+\delta_{1} B a l_{t}+\delta_{2} \Delta x_{t}
\end{aligned}
$$

and a circumflex denotes that unknown parameters are replaced by their maximum likelihood estimates. Diagnostic tests can then be carried out on the generalised residuals; e.g., see Machin \& Stewart (1990). We test their normality (using a Jarque-Bera test) and their cross-sectional independence. We use the cross-sectional independence test developed by Hsiao et al. (2007) for use with nonlinear panel data models. The CD test statistic is based on the average (across firms $i=1, \ldots, N_{t}$ ) pair-wise generalised residual correlation coefficient. Under the null of cross-sectional independence, the CD statistic tends to a standard normal variate. The econometric analysis and the results of these tests are presented in Section 5.1.1. 


\subsection{Hypothesis testing: signal and noise}

We examine a range of possible restrictions on the parameters of equation (7). In Section 5.1 .2 we explore the significance of individual coefficients and in section 5.1 .3 we test the joint hypotheses which we now describe.

For the qualitative survey data to be clearly "useful", or contain a signal about the quantitative data, the MPI turnover growth terms in (7) have to be statistically significant. Conversely, when there is no informational content to the ITS responses, and they constitute only noise, we should expect the following hypothesis to hold:

$$
H_{0}^{1}: \gamma_{1}=\gamma_{2}=\cdots=\gamma_{12}=0
$$

Moreover, when a firm does, as instructed by the CBI, base its qualitative reply on its trend growth over the last three (previously four) months, we might expect lags in (7), beyond the horizon of interest (namely three/four months), to be statistically insignificant:

$$
H_{0}^{2}: \gamma_{k+1}=0 \text { and } \lambda_{k}^{(j)}=0 \text {, for all } k>4, j=0 \text { and } 2 .
$$

But rejection of $H_{0}^{2}$ need not imply that firms do not follow the instructions of the CBI by looking further into the past than asked when reporting their trend output growth. When fluctuations in turnover are substantially accounted for by fluctuations in stocks, as appendix A.3 explains, the statistical significance of lagged values for $\Delta x_{i, t}$ may reflect the endogeneity of turnover growth rather than the direct reaction of ITS respondents to lagged $\Delta x_{i, t}$. But irrespective of whether turnover growth is exogenous or not, when firms follow the CBI's instructions we should expect the lagged qualitative responses $y_{i, t}^{(j)}(j=0$ and $j=2$ ) to be statistically insignificant at lags beyond the horizon deemed of interest by the CBI (i.e., three or four months). At shorter lags, say $y_{i, t-1}^{(j)}$, we should expect dependence even when firms follow the CBI's instructions since $y_{i, t}^{*}$ and $y_{i, t-1}^{(j)}$ overlap given that they refer, respectively, to growth over the last three/four months and growth one month ago relative to four/five months ago.

When $H_{0}^{2}$ is rejected we isolate the cause by breaking (15) down into two constituent tests:

$$
H_{0}^{3}: \gamma_{k+1}=0, \text { for all } k>4
$$

and

$$
H_{0}^{4}: \lambda_{k}^{(j)}=0, \text { for all } k>4, j=0 \text { and } 2
$$


where $H_{0}^{3}$ tests whether firms base their qualitative response on quantitative information 'too' far back into the past and $H_{0}^{4}$ tests if there is 'too' much (relative to the CBI's question) inertia in firms' qualitative responses. Due to the change in the reference period of the ITS question over our sampling period, we also consider variants of these tests for $k>3$. Denote the tests for $k>3, H_{0}^{2 a}, H_{0}^{3 a}$ and $H_{0}^{4 a}$, and those for $k>4, H_{0}^{2 b}, H_{0}^{3 b}$ and $H_{0}^{4 b}$.

Finally we explore the possibility that the lag in turnover growth should be longer than the eleven months set out in equation (7). We do this as a variable addition test, adding addition terms to equation (7): $\gamma_{13} \Delta x_{i, t-12}+\gamma_{14} \Delta x_{i, t-13}+\gamma_{15} \Delta x_{i, t-14}+\gamma_{16} \Delta x_{i, t-15}$, and testing, relative to the unrestricted equation, $H_{0}^{5}$ where:

$$
H_{0}^{5}: \gamma_{k+1}=0, \text { for all } k>12
$$

\subsection{A quantitative indicator of firm-level growth constructed from the ITS}

To assess whether any signal in the ITS data has value to economists we examine the ability of the qualitative data to predict (or nowcast) the firm-level quantitative data. This involves inverting the probit models, via Bayes' Theorem (see also Mitchell et al. (2006)), and constructing an early indicator of the quantitative data based on the qualitative data. Particular interest rests on how useful the ITS data published at time $t$ are at predicting (or, more accurately, nowcasting) the MPI data at time $t, \Delta x_{i, t}$. But given the possibility that the ITS data tell us not just about $\Delta x_{i, t}$, but lags of $\Delta x_{i, t}$, we construct indicators of $\Delta x_{i, t-k}(k=0,1,2, \ldots)$.

Let $j_{i, t},\left(j_{i, t}=0,1,2\right)$, denote the qualitative survey response of firm $i$ at time $t$. Let $f\left(\Delta x_{i, t}, \cdots, \Delta x_{i, t-k} \mid\left\{\Delta x_{i, \tau}\right\}_{\tau=1}^{t-k-1}\right)$ denote the prior conditional density for the quantitative data, constructed without reference to the qualitative data. Given the likely correlation of $\Delta x_{i, t}, \cdots, \Delta x_{i, t-k}$, this multivariate conditional density is assumed to follow a multivariate normal distribution

$$
f\left(\Delta x_{i, t}, \cdots, \Delta x_{i, t-k} \mid\left\{\Delta x_{i, \tau}\right\}_{\tau=1}^{t-k-1}\right) \sim N(\mu, \Sigma) .
$$

We need to work out the density function of $\Delta x_{i, t-k}$ conditional on the firms' observed qualitative survey responses at time $t$ and earlier, and conditional on lagged quantitative information $\left\{\Delta x_{i, \tau}\right\}_{\tau=1}^{t-k-1}$, and the macro-economic data $\left(B a l_{t}\right.$ and $\left.x_{t}\right)$ which, to ease notation, we do not condition on explicitly below but take as read. We denote this 
density function $f\left(\Delta x_{i, t-k} \mid\left\{j_{i, \tau}\right\}_{\tau=1}^{t},\left\{\Delta x_{i, \tau}\right\}_{\tau=1}^{t-k-1}\right)$. Our indicator, $D_{i, t-k}(k=0,1,2, \ldots)$, under squared error loss, is then given as:

$$
\begin{aligned}
D_{i, t-k} & =E\left(\Delta x_{i, t-k} \mid\left\{j_{i, \tau}\right\}_{\tau=1}^{t},\left\{\Delta x_{i, \tau}\right\}_{\tau=1}^{t-k-1}\right) \\
D_{i, t-k} & =\int_{-\infty}^{\infty} \Delta x_{i, t-k} f\left(\Delta x_{i, t-k} \mid\left\{j_{i, \tau}\right\}_{\tau=1}^{t},\left\{\Delta x_{i, \tau}\right\}_{\tau=1}^{t-k-1}\right) d \Delta x_{i, t-k} .
\end{aligned}
$$

This is the expectation of firm-level growth at time $t-k$, conditional on the qualitative data up to and including time $t$ but quantitative data only up to and including time $t-k-1$, reflecting the lagged availability of the MPI. The value of the qualitative survey data rests on comparison of $D_{i, t-k}$ against the autoregressive benchmark indicator $E\left(\Delta x_{i, t-k} \mid\left\{\Delta x_{i, \tau}\right\}_{\tau=1}^{t-k-1}\right)$.

Bayes' theorem states that

$$
f\left(\Delta x_{i, t-k} \mid\left\{j_{i, \tau}\right\}_{\tau=1}^{t},\left\{\Delta x_{i, \tau}\right\}_{\tau=1}^{t-k-1}\right)=\frac{P\left(j_{i, t}, \Delta x_{i, t-k} \mid\left\{j_{i, \tau}\right\}_{\tau=1}^{t-1},\left\{\Delta x_{i, \tau}\right\}_{\tau=1}^{t-k-1}\right)}{P\left(j_{i, t} \mid\left\{j_{i, \tau}\right\}_{\tau=1}^{t-1},\left\{\Delta x_{i, \tau}\right\}_{\tau=1}^{t-k-1}\right)}
$$

where

$$
\begin{gathered}
P\left(j_{i, t}, \Delta x_{i, t-k} \mid\left\{j_{i, \tau}\right\}_{\tau=1}^{t-1},\left\{\Delta x_{i, \tau}\right\}_{\tau=1}^{t-k-1}\right)= \\
=\int_{-\infty}^{\infty} \cdots \int_{-\infty}^{\infty} b f\left(\Delta x_{i, t}, \cdots, \Delta x_{i, t-k} \mid\left\{\Delta x_{i, \tau}\right\}_{\tau=1}^{t-k-1}\right) d \Delta x_{i, t} \cdots d \Delta x_{i, t-k+1},
\end{gathered}
$$

and $b$ integrates out the random effect $\alpha_{i}$

$$
b=\left(\int_{-\infty}^{\infty} P\left(j_{i, t} \mid\left\{j_{i, \tau}\right\}_{\tau=1}^{t-1},\left\{\Delta x_{i, \tau}\right\}_{\tau=1}^{t}, \alpha_{i}\right) f\left(\alpha_{i}\right) d \alpha_{i}\right) .
$$

The denominator of (22) involves integrating $\Delta x_{i, t-k}$ out from (23). Note that when $k=0$, since future values of the quantitative data do not enter the probit models, (23) reduces to

$$
P\left(j_{i, t}, \Delta x_{i, t} \mid\left\{j_{i, \tau}\right\}_{\tau=1}^{t-1},\left\{\Delta x_{i, \tau}\right\}_{\tau=1}^{t-1}\right)=\int_{-\infty}^{\infty} b f\left(\Delta x_{i, t} \mid\left\{\Delta x_{i, \tau}\right\}_{\tau=1}^{t-1}\right) d \Delta x_{i, t} .
$$

Given $f\left(\Delta x_{i, t-k} \mid\left\{j_{i, \tau}\right\}_{\tau=1}^{t},\left\{\Delta x_{i, \tau}\right\}_{\tau=1}^{t-k-1}\right)$, with $\mu$ and $\Sigma$ estimated by least squares, all of 
the above integrals may be calculated by numerical evaluation.

Estimators $\widehat{P}\left(j_{i, t} \mid\left\{j_{i, \tau}\right\}_{\tau=1}^{t-1},\left\{\Delta x_{i, \tau}\right\}_{\tau=1}^{t}, \alpha_{i}\right)$ for $P\left(j_{i, t} \mid\left\{j_{i, \tau}\right\}_{\tau=1}^{t-1},\left\{\Delta x_{i, \tau}\right\}_{\tau=1}^{t}, \alpha_{i}\right)$ are given by substitution of the estimators $\widehat{\sigma}_{\alpha}^{2}, \hat{\mu}_{j}(j=0, \ldots, 3), \widehat{\gamma}_{l}(l=0, \ldots, 11), \widehat{\lambda}_{m}^{j}(m=1, \ldots, 12$, $j=0$ and 2), $\widehat{\delta}_{1}$ and $\widehat{\delta}_{2}$, in 10 . Hence, a feasible empirical Bayes estimator

$$
D_{i, t-k}=\widehat{E}\left(\Delta x_{i, t-k} \mid\left\{j_{i, \tau}\right\}_{\tau=1}^{t},\left\{\Delta x_{i, \tau}\right\}_{\tau=1}^{t-k-1}\right)
$$

may be obtained by numerical evaluation. The impact of the use of plug-in (estimated) parameters, instead of priors for these parameters, is expected to be small when the likelihood dominates the prior for the parameters, which it does in large samples and/or when the priors are vague. Deely \& Lindley (1981) show that the empirical Bayes predictor is a first order approximation to the Bayes predictor.

\section{Empirical Results}

\subsection{Signal or noise?}

\subsubsection{Probit Equations}

Dynamic ordered probit models, (7) and (9), with $p=1, \ldots, 11$ lags of $y_{i, t-1}^{(j)}(j=0$ and $j=2$ ) and $\Delta x_{i, t}$ are estimated on the sub-panel of 96 firms with at least 12 consecutive matched responses. Use of this sub-panel is necessary in order to estimate dynamic models which allow firms' qualitative responses to be potentially affected by events and their own qualitative responses up to a year ago; below in Section 5.1.4 we consider how robust results are to use of this 96 firm sub-panel. The Bayesian Information Criterion (BIC) is then used to select the preferred number of lags, $p$. This process is different from identifying individually insignificant coefficients, an issue we discuss later, since those associated with very short lags will not be restricted to zero if longer lags play a statistically significant role. Table 5 reports the estimation results for this preferred model. The model is estimated with both the raw data, prior to outlier treatment, and the Winsorised data. The estimated coefficients are closer to zero for the raw data than the Winsorised data; as expected, Winsorisation appears to remove noise from the MPI data and delivers coefficient estimates with higher (robust) $t$-statistics as well as a model with better overall fit, evidenced by a higher value for the maximised $\log$-likelihood $(\ln L)$ and, in turn, a lower value for the BIC in Table 5. This gives a statistical reason for preferring to work with the results from the Winsorised data and henceforth we confine 
attention to the results from these.

In Table 5 we see that the quantitative data, $\Delta x_{i, t}$, are statistically insignificant at time $t$ and it is lagged values of $\Delta x_{i, t}$ that help explain the qualitative data. The lagged qualitative data also play an important role with larger $t$-statistics on the coefficients of $y_{i, t-1}^{(j)}(j=0$ and $j=2)$ than on $\Delta x_{i, t-1}$ and $\Delta x_{i, t-2}$. We cannot reject the restriction that $\sigma_{\alpha}^{2}=0$ and so confine attention to the pooled dynamic ordered probit model. Seasonal, time, sectoral and size dummies were included in the probit model but found to be statistically insignificant. We cannot reject the hypothesis that seasonal and time dummies are jointly insignificant with a $p$-value of $71 \%$. Sectoral and size dummies are also proved to be jointly insignificant with a $p$-value of $6.5 \%$. The macro-economic data, $\Delta x_{t}$, were found to be insignificant with a $p$-value of $21.6 \%$ and were excluded from the model. However, the balance statistic, as shown in the table, was statistically significant with a $p$-value of $0 \%$. The diagnostic statistics in Table 5 also indicate that the generalised residuals are free from non-normality and cross-sectional dependence lending support to our modelling approach.

\subsubsection{Significance of Individual Lags}

To provide further indication of the relationship between the ITS and MPI data we now turn to the unrestricted model based on estimation of the dynamic ordered probit models,

(7) and (9), with $p=11$ lags of $y_{i, t-1}^{(0)}, y_{i, t-1}^{(2)}$ and $\Delta x_{i, t}$. In the upper panel of Figure 2 we plot the $t$ - statistics of the estimated coefficients on $\Delta x_{i, t}$ to $\Delta x_{i, t-11}$. The $t$-statistics do not, of course, necessarily represent the relative importance of the different parameters. However, since the standard errors of the different parameters whose t-statistics are shown in these two graphs are very similar at different lags, the profiles shown in Figure 2 also represent the relative importance of the different lags.

The top panel of Figure 2 shows that the $t$-values for the short lags of $\Delta x_{i, t-k}$ are often greater than \pm 1.96 , the $95 \%$ critical value. But, as in Table 5 , one can reject the view that the qualitative survey data provide a good coincident indicator of growth, since the estimated coefficient on $\Delta x_{i, t-k}$ has a $t$-value of only -0.4 at $k=0$.

Figure 2 shows that growth as reported in the firm-level qualitative survey responds most strongly to monthly growth one and two months earlier $(k=1$ and $k=2)$ and not contemporaneously. The peak influence is $2-3$ months ago $(k=2)$, with the signal weakening thereafter. However, the $t$-values remain greater than 1.96 up to 5-6 months 
Table 5: Estimation output for the parsimonious ordered probit model

\begin{tabular}{l|c|c|c|c}
\hline \multicolumn{1}{c|}{ Explanatory variables } & \multicolumn{2}{|c|}{ Estimated coeff. } & \multicolumn{2}{c}{ Robust $t$-stat } \\
\hline & raw data & $\begin{array}{c}\text { Winsorised } \\
\text { data }\end{array}$ & raw data & $\begin{array}{c}\text { Winsorised } \\
\text { data }\end{array}$ \\
\hline$\Delta x_{i, t}$ & -0.0254 & 0.0696 & -0.36 & -0.43 \\
$\Delta x_{i, t-1}$ & 0.2070 & 0.6794 & 2.36 & 3.76 \\
$\Delta x_{i, t-2}$ & 0.2960 & 0.7590 & 3.81 & 4.34 \\
$y_{i, t-1}^{u}$ & 1.0177 & 1.0339 & 9.43 & 9.53 \\
$y_{i, t-1}^{d}$ & -0.8639 & -0.8440 & -7.75 & -7.54 \\
$y_{i, t-2}^{u}$ & 0.6023 & 0.6176 & 5.44 & 5.55 \\
$y_{i, t-2}^{d}$ & -0.4520 & -0.4719 & -4.27 & -4.43 \\
Bal & 0.0105 & 0.0108 & 2.83 & 2.93 \\
& & & & \\
lower threshold $\mu_{1}$ & -0.8495 & -0.8496 & -13.01 & -13.00 \\
upper threshold $\mu_{2}$ & 0.9109 & 0.9266 & 14.08 & 14.15 \\
\hline Number of obs $\left(\sum \sum_{i=1}^{N} T_{i}\right)$ & & & 1033 & 1033 \\
$N$ & & & 96 & 96 \\
Wald chi2 $(8)$ & & & 412.68 & 421.76 \\
Prob $>$ chi2 & & & 0.00 & 0.00 \\
Pseudo $R^{2}$ & & & 0.2631 & 0.2687 \\
ln $L$ & & & -811.642 & -805.477 \\
BIC & & & 0.56 & 1680.36 \\
CD test $p$-value & & & 0.64 \\
Normality test $p$-value & & & 0.17 & 0.24 \\
p-value: $\sigma_{\alpha}^{2}=0$ & & & &
\end{tabular}

Note: Estimated using the raw MPI data and MPI data with $5 \%$ Winsorisation. Wald chi2(8) is a Wald test for insignificance of the coefficients on all the explanatory variables and Prob $>$ chi 2 is the associated p-value. $\ln L$ is the maximised value of the log-likelihood; BIC is the Bayesian Information Criterion; CD is the cross-sectional independence test. 
ago $(k=5)$, and are less than 1.96 thereafter except for a spike at $k=11$. As discussed above this conclusion may be because of the interaction between output and sales rather than because firms look further back than the CBI requests. This finding apparently contradicts the earlier model which, in the light of the BIC was restricted to two lags. However, the BIC is known for its property of leading to parsimonious models and these are, in turn, widely found to be robust in modelling applications; see Clements \& Hendry (1998).

The firm-level results are given alongside those from an analogous auto-regressive distributed-lag (ARDL) model estimated on aggregated (macroeconomic) data. ARDL models are used widely in macroeconomics to model dynamic relationships; e.g., see Hendry et al. (1984) and, as mentioned above, Pesaran (1997). The ARDL model is estimated using manufacturing output growth, $\Delta x_{t}$, calculated from the manufacturing component of the IoP (which shows output and not sales) and the balance statistic, $\mathrm{Bal}_{t}$, from the ITS described in the account of equation (9). Consistent with our view that the qualitative survey data provide noisy estimates of the official data (i.e., the measurement error is in the qualitative survey data rather than the official data), the ARDL model is estimated by OLS with the qualitative data (the balance statistic $B a l_{t}$ ) as the regressand. As with (7), again twelve lags of the regressor $\left(\Delta x_{t}\right)$ and regressand are considered. The balance statistic is available from January 1985 and, after allowing for lags, our estimation period is therefore January 1986 to December 2007. We tested the stability of the regression by estimating over two sub-periods, January 1986 to December 1997 and January 1998 to December 2007; we cannot reject the hypothesis of parameter stability on the basis of the Chow test with $F(25,239)=0.86, p=66 \%$.

The peak $t$-statistic in Figure 2 for the macroeconomic data is at $k=4$, rather than $k=2$. This indicates that the macroeconomic signal lags the firm-level signal.

In the upper panel of Figure 3 we show similar results for the $t$-statistics associated with the estimated coefficients on the lagged dummy variables, $y_{i, t-k}^{(j)} k=1$ to $12(j=0,2)$. We can see that the significance of the lags falls off after $k=2$, and the pattern is similar for both firms reporting down $j=0$ and reporting up, $j=2$. In part, as discussed, this is due to the overlapping nature of the responses rather than genuine state dependence. However with $k=10$ the coefficient $\lambda_{10}^{(2)}$ has a $t$ - ratio greater than 2 . The lower panel plots the analogous $t$ - statistics on the lags of the balance statistic from the macro-economic regression. There is, of course, only one parameter rather than two associated with the lags in this case since the balance statistic replaces the two dummy variables. These $t$-statistics show somewhat greater persistence. For a lag of 2 the $t$ - statistic is only 1.6 

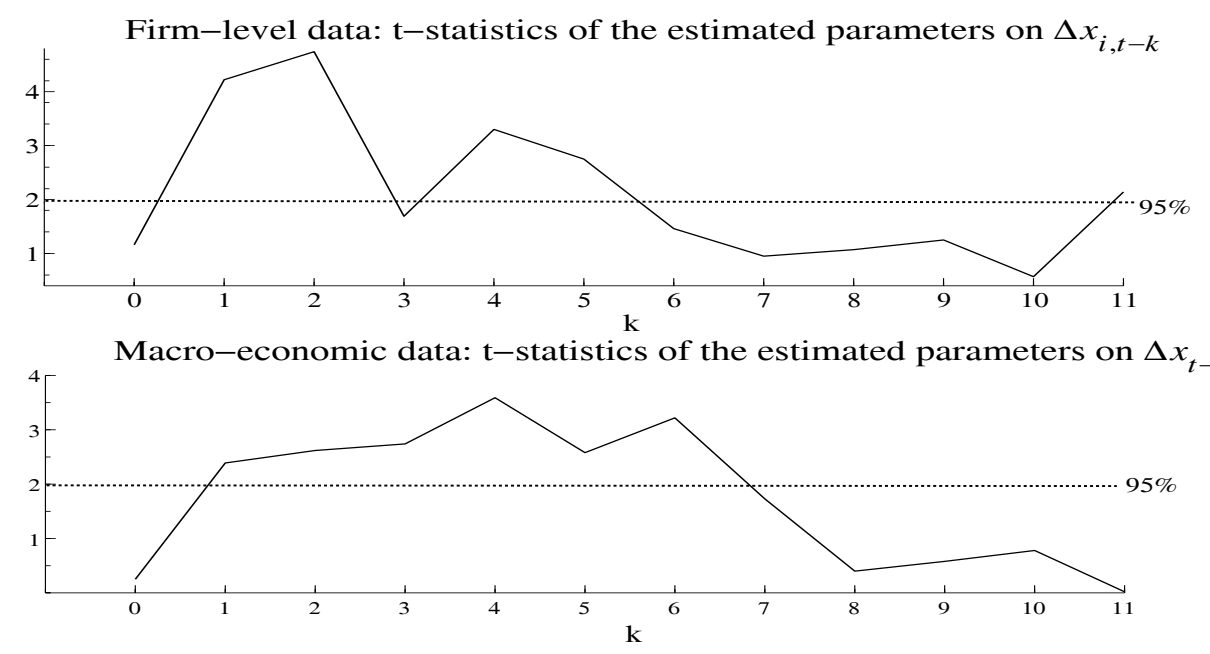

Figure 2: t-statistics for the estimated coefficients on the rolling 1-month MPI turnover growth rates in the firm-level data (top panel) and macroeconomic data (bottom panel). $95 \%$ critical values are \pm 1.96 for both panels

but it rises above two with a lag of $k=3$, and is also above two (in absolute value) with lags of $k=7$ and $k=9$.

\subsubsection{Joint Hypothesis Tests}

An alternative perspective is offered by testing the range of hypotheses about joint significance of the coefficients which we discussed in Section 4.1. Table 6 summarises the results of these tests, again based on estimation of the unrestricted dynamic ordered probit models, (7) and (9), with eleven lags.

The $p$-values in Table 6 indicate that the hypothesis of noise, $H_{0}^{1}$, is clearly rejected with a $p$-value less than $1 \%$ for both the firm-level and macroeconomic data. The ITS data are plainly related to the responses the same firms give to the MPI. But since it is unclear how exactly firms interpret the ITS question, the remaining hypothesis tests in Table 6 shed more light on what the respondents actually had in mind. The failure for the firm-level data to reject $H_{0}^{2 a}$ or $H_{0}^{2 b}$ (with $p$-values of $17 \%$ and $20 \%$ ) indicates that firms do appear to follow the CBI's instructions 'quite' closely by basing their qualitative responses on MPI growth over the last 3 to 4 months; we discuss this further below. Comparison of the firm-level and macroeconomic results reveals that there is also a signal 


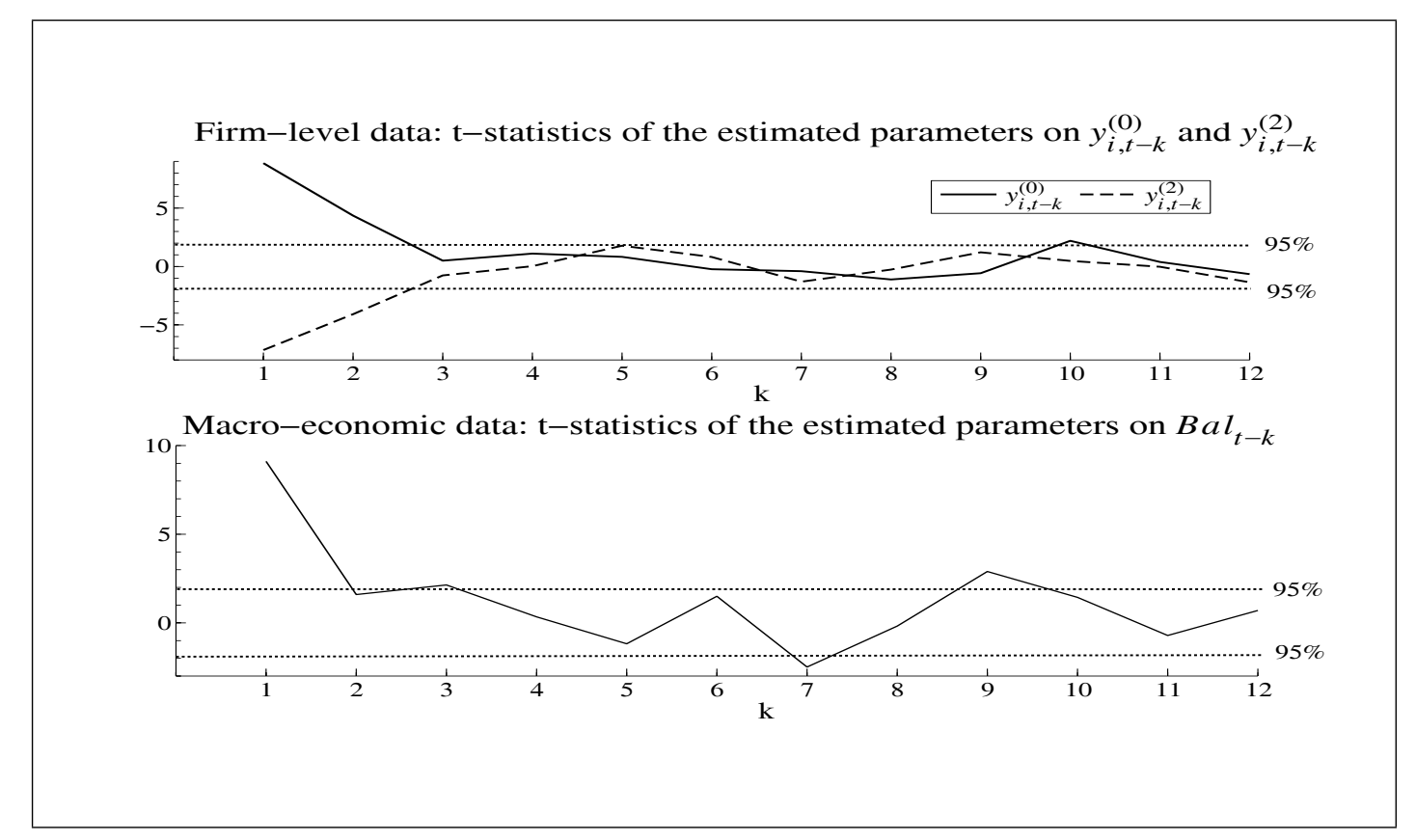

Figure 3: $t$-statistics for the dummy variables for the lagged ITS responses in the firmlevel data (top panel) and for the estimated coefficients on lags of the balance statistic in the macroeconomic data (bottom panel): $95 \%$ critical values are \pm 1.96 for both panels

Table 6: Signal or noise? p-values for the hypothesis tests on the firm-level data and the macroeconomic data

\begin{tabular}{c|c|c}
\hline Likelihood ratio tests: & Firm-Level & Macro \\
\hline$H_{0}^{1}$ & 0.0001 & 0.0012 \\
$H_{0}^{2 a}$ & 0.1672 & 0.0003 \\
$H_{0}^{2 b}$ & 0.1975 & 0.0025 \\
$H_{0}^{3 a}$ & 0.0417 & 0.0038 \\
$H_{0}^{3 b}$ & 0.0749 & 0.0524 \\
$H_{0}^{4 a}$ & 0.5004 & 0.0185 \\
$H_{0}^{4 b}$ & 0.4777 & 0.0134 \\
$H_{0}^{5}$ & 0.4986 & 0.5673 \\
\hline
\end{tabular}

Notes: $H_{0}^{1}: \gamma_{1}=\gamma_{2}=\cdots=\gamma_{12}=0 ; H_{0}^{2}: \gamma_{k+1}=0$ and $\lambda_{k}^{(j)}=0$, for all $k>4, j=0$ and $2 ; H_{0}^{3}: \gamma_{k+1}=0$, for all $k>4$; $H_{0}^{4}: \lambda_{k}^{(j)}=0$, for all $k>4, j=0$ and $2 ; H_{0}^{5}: \gamma_{k+1}=0$, for all $k>12$. Due to the change in the reference period of the ITS question over our sampling period, $H_{0}^{2 a}, H_{0}^{3 a}$ and $H_{0}^{4 a}$ denote the tests for $k>3$ and $H_{0}^{2 b}, H_{0}^{3 b}$ and $H_{0}^{4 b}$ denote those for $k>4$. The hypothesis tests are defined fully in Section 4.1. 
in the ITS data at the aggregate-level, since $H_{0}^{1}$ is again rejected, with a $p$-value of less than $1 \%$.

Exploration of hypothesis $H_{0}^{3 a}$ again draws attention to a conflict between the results of the hypothesis test and the use of the BIC criterion to specify the equation of Table 5 . We can see that we reject the hypothesis that the coefficients on all lags of $\Delta x_{i, t-k}$ longer than three months can be set to zero on the basis of this hypothesis test, with a $p$-value of $4 \%$.

Nevertheless, comparison of the $p$-values for $H_{0}^{3 a}$ and $H_{0}^{3 b}$ in Table 6 indicates that the signal from the qualitative data does weaken when related to longer lags of $\Delta x_{i, t-k}$; the $p$-value rises from $4 \%$, for $H_{0}^{3 a}$ when $k>3$, to $7 \%$ for $H_{0}^{3 b}$ when $k>4$. But these $p$-values are lower than those for $H_{0}^{4 a}$ and $H_{0}^{4 b}$, which are around $50 \%$. This indicates that the aforementioned support for the view that firms look no further than 3 or 4 months into the past (seen in $p$-values of $17 \%$ and $20 \%$ for $H_{0}^{2 a}$ or $H_{0}^{2 b}$ ) is misleading. Once the qualitative data at longer lags are excluded we find that rejection of the view that firms do not look back further than three/four months is marginal.

In the macro equation we reject, with a $p$-value of $0.38 \%$, the hypothesis $H_{0}^{3 a}: \gamma_{t-k}=0$, for all $k>3$ much more firmly. This implies that the balance statistic, more obviously than the firm-level responses, tells us about growth in the economy further back into the past than just the last three months. However, as with the firm-level data, this signal does appear to weaken thereafter, since one can only reject the null hypothesis that $\gamma_{t-k}=0$, for all $k>4$, with a $p$-value of $5.24 \%$. Long lags of the balance statistic are significant in the macro equation much more than in the firm-level equation since $H_{0}^{4 a}$ and $H_{0}^{4 b}$ are firmly rejected in the latter but easily not rejected in the former case. Finally there is no suggestion that very long lags of $\Delta x_{i, t}$ or $\Delta x_{t}$ are relevant; we cannot reject $H_{0}^{5}$ with $p$-values of $49.9 \%$ with the firm-level data and $56.7 \%$ with the macro data.

Thus our overall conclusion is that it is marginal whether one can accept the hypothesis that firms responses to the qualitative survey reflect only the quantitative growth rates that they report within the last three/four months. The outcome of the test depends on the precise nature of the hypothesis tested. But, since statistical significance of the longer $\operatorname{lag}(\mathrm{s})$ may be due to the fact that firms report sales rather than output to the MPI, one cannot firmly conclude that firms report output movements over a period longer than the CBI requests. 


\subsubsection{Robustness of the signal}

Only those firms with at least twelve consecutive observations (or thirteen in the level of turnover) are included in the model, (7) and (9). This means our sample dropped from the 807 different firms seen in Table 1 to 96 firms. To provide some check on how reliable results from this sub-panel are we undertake two exercises. First we estimate variants of (7) and (9) that restrict the specification so it is akin to testing the significance of the partial (polyserial) correlation between the ITS and MPI data for a given value of $k$; see Olsson et al. (1982). The implied restrictions, while rejected by the data (as shown by Table 6) mean that a larger sub-panel of the 807 matched firms can be considered. Importantly, and reassuringly, we find that the main results from Tables 5 and 6 are robust to imposition of these restrictions. Secondly, we undertake tests for sample selection.

Restricted specifications The restricted specifications considered involve, separately for each $k$, relating $y_{i, t}^{*}$ to $z_{i, t}^{(k)}=\ln x_{i, t}-\ln x_{i, t-k}$ and $\Delta x_{i, t-k+1}=\ln x_{i, t-k+1}-\ln x_{i, t-k}$ $(k=1, \cdots, 12)$ and then estimating an ordered probit model for each of the twelve values of $z_{i, t}^{(k)}$ and $\Delta x_{i, t-k+1}$. The balance statistic, $B a l_{t}$, continues to be included in these specifications. As in Table 5 the coefficient on $\mathrm{Bal}_{t}$ is statistically significant in all the models. The lowest of the twelve values for the $t$-statistics associated with the model using $z_{i, t}^{(k)}$ is 5.76 while for $\Delta x_{i, t-k+1}$ it is 6.16 giving $p$-values of $0 \%$ to three decimal places in all cases. The significance of $z_{i, t}^{(k)}$ or $\Delta x_{i, t-k+1}$ in these models can be interpreted as measuring the degree of (partial) association between the ITS and MPI data. Were it not for the presence of the balance statistic this would amount to testing the significance of the unconditional (polyserial) correlation coefficient.

Figure 4 reports the $t$-statistics of the estimated coefficients on the MPI turnover growth rates from these ordered probit models. A histogram is also presented indicating the number of observations, pooled across firms and time, used for estimation at a given value of $k$. This indicates that the sample size for these restricted models is much larger than in Table 5 ,

Figure 4 indicates that the $t$-statistics of $z_{i, t}^{(k)}$ increase with $k$, meaning the relationship between the qualitative and quantitative data becomes increasingly strong as we look further back into the past. This implies that the qualitative data are indeed a lagging indicator as Tables 5 and 6 suggest. This is to be expected given that the ITS question asks about growth over the last three/four months, although as discussed it is unclear both how exactly firms interpret this and what effect measurement error has. Like the 


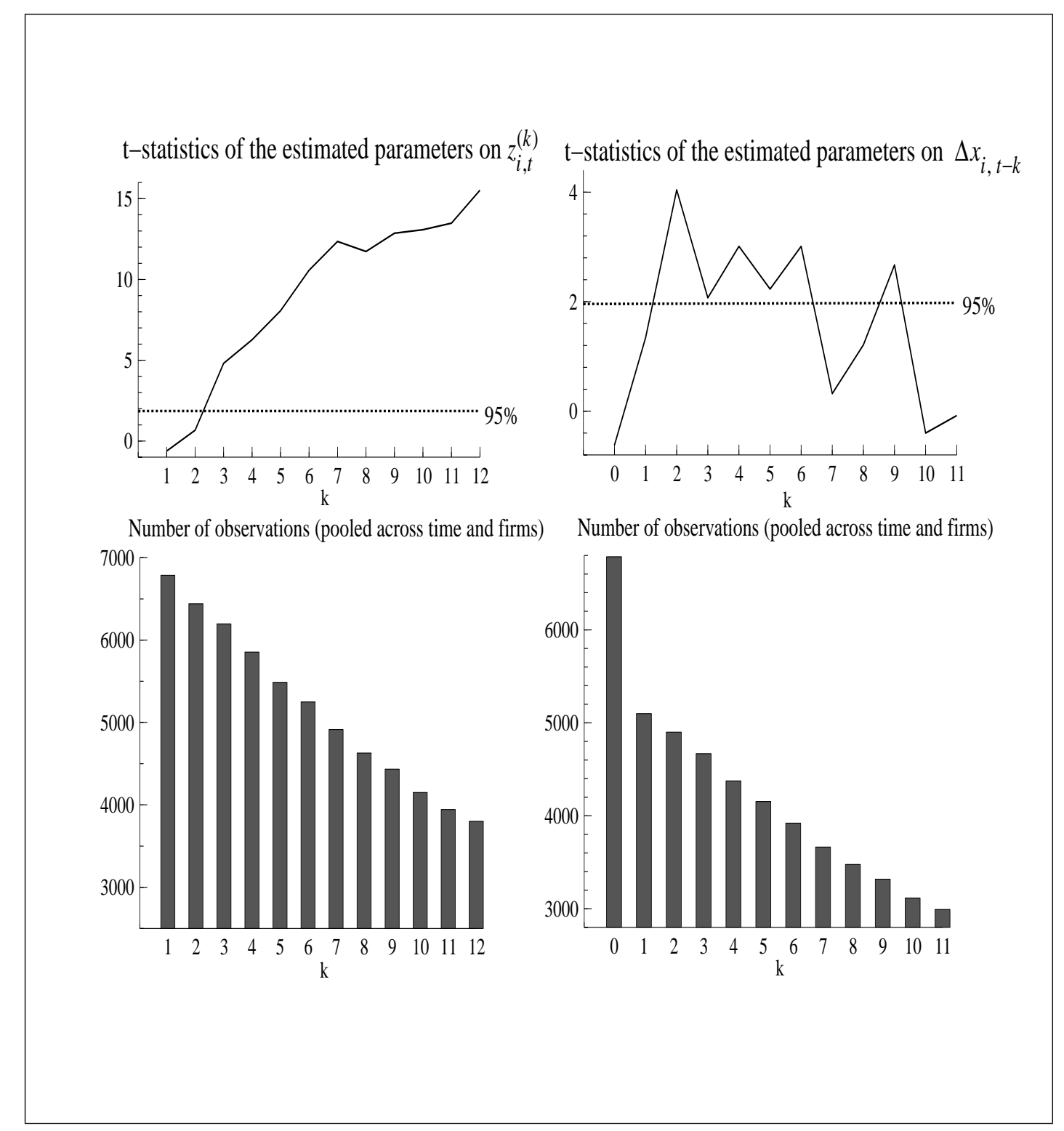

Figure 4: t-statistics of estimated coefficients on MPI turnover growth rates in an ordered probit model explaining the ITS data, plus a histogram indicating the sample size. 95\% confidence values are \pm 1.96 . The left-hand panels show results for $z_{i, t}^{(k)}=\ln x_{i, t}-\ln x_{i, t-k}$ and the right-hand panels for $\Delta x_{i, t-k}=\ln x_{i, t-k}-\ln x_{i, t-k-1}$. 
top panel of Figure 2, the right-hand-side of Figure 4 indicates that again the coefficients on $\Delta x_{i, t-k}$ become statistically significant, at a $95 \%$ level of significance, only when $k \geq 2$. In other words, the partial analysis also suggests that the firm-level ITS data at time $t$ do not contain a statistically significant signal about growth in the last month, but about growth two months ago relative to three months ago. As in Figure 2 as $k$ increases the significance of the coefficients on $\Delta x_{i, t-k}$ decreases. But the signal is still statistically significant (at 95\%) for growth up to six, relative to seven, months ago. The signal is in general insignificant after seven months, except at nine relative to ten months. This somewhat strengthens our earlier conclusion and leads us to conclude that, on balance, there is statistical evidence to indicate that MPI data more than three or four months ago do influence firms when replying to the ITS, although, as we have noted, this could be a result of the endogeneity of the MPI data rather than a direct reaction to lagged MPI data. In any case, the signal does weaken the further back into the past one looks. We also found (and report in the working paper version of this paper) that when we estimated similar models at the sectoral level or for different sized-firms these different firms displayed a relationship with the quantitative data similar to that seen in Figure 4.

Interestingly, Figure 5 shows that a similar relationship holds at the macroeconomic level (i.e., for the manufacturing sector as a whole and where the issue of the difference between sales and output does not arise). The figure plots the $t$-statistics associated with the estimated coefficient on manufacturing output growth, $\Delta x_{t-k}$, obtained from an OLS regression, separately for each $k$, of the balance statistic $\left(B a l_{t}\right)$ on manufacturing output growth $\left(\Delta x_{t-k}\right)$ using data from January 1985 to December 2007.

As with Figure 4 , Figure 5 plots the $t$-statistics on the $k$-month growth rate, now in the top panel, and the rolling 1-month growth rate, in the bottom panel. Looking at the top panel, as with Figure 4 , it is seen that the coefficient on contemporaneous growth $\left(\Delta x_{t}\right)$ is not statistically significant and the $t$-statistics rise steadily above the $95 \%$ significance level as the length of the interval considered increases. The bottom panel of Figure 5 shows that the peak signal arises from monthly growth lagged six months. The peak signal with the firm-level data was a little earlier, with a lag of two months. But both the firm-level and macroeconomic evidence suggest that the qualitative survey data do not provide a good coincident indicator of growth.

Tests for sample selection To provide a further indication of how representative results from the 96 firm sub-panel are, we make use of the variable addition tests of Verbeek \& Nijman (1992). These involve adding test variables to the random effects 

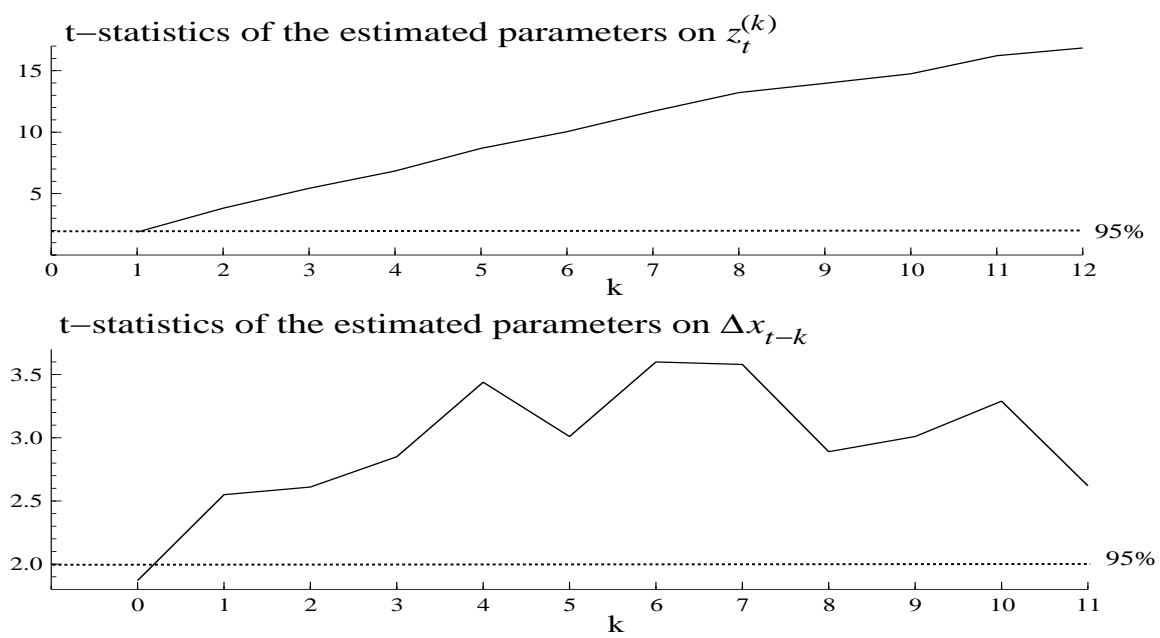

Figure 5: $t$-statistics indicating the strength of the relationship between the ITS and MPI data at the macroeconomic level

ordered probit model considered in Table 5. The first test variable is a count variable characterising the number of times a firm is present in the matched dataset (summarised in Figure 1) across the 60 months. When there is no sample selection we should not expect firms' qualitative responses to be associated with the number of times a firm is present in the matched dataset. The second test variable is a count variable characterising the number of times a firm is present in the ITS sample across the 60 months. Again if there is no sample selection this variable should be statistically insignificant. We find $p$-values of $89.5 \%$ and $35 \%$ for the first and second count variables, respectively. These results support the view that the results from the 96 firms are free from sample selection and there is no tendency for these 96 firms to be either particularly good or bad at replying to the ITS although there is obviously a risk that it is lack of power rather than the genuine absence of selection effects which explains our results.

We also re-estimated the parsimonious ordered probit model in Table 5 but, since we no longer test this model against more general alternatives which reduced the number of firms available to 96, let an additional 285 firms enter the sample. A Hausman test cannot reject the null of equivalence between the two estimators at $95 \%$ [the $p$-value was $10.7 \%$ ], lending further support to the view that the results reported in Tables 5 and 6 are free from sample selection biases. 


\subsection{The ITS as an early indicator of the MPI}

We assess the predictive power of the indicator $D_{i, t-k}$ given in $(26)$ based on the in-sample fit of the probit models. An out-of-sample analysis, which involves splitting the sample, $T$, in two and estimating the models recursively, is not sensible in this application given that $T=60$. Moreover, in-sample tests of predictability have been found to have greater power than out-of-sample tests; see Inoue \& Kilian (2004).

Table 7 summarises the performance of $D_{i, t-k}$ by reporting the correlation and Root Mean Squared Error (RMSE) of the indicator, pooled across firms and time, against $\Delta x_{i, t-k}$. Performance of $D_{i, t-k}$ is distinguished according to the prior density, $f\left(\Delta x_{i, t}, \cdots, \Delta x_{i, t-k} \mid\left\{\Delta x_{i, \tau}\right\}_{\tau=1}^{t-k-1}\right)$, chosen to model the MPI data; for robustness, we consider two choices, an AR for each element of $\left(\Delta x_{i, t}, \cdots, \Delta x_{i, t-k}\right)^{\prime}$ with $p=k+1, \ldots, 11$ lags and an AR with just a single lag. The latter might be expected to benefit from the generally favourable effects of parsimony on forecasting performance; see Clements \& Hendry (1998). The final two columns of Table 7 summarise the performance of fitted values from these two models. Since $D_{i, t}$ is the mean of the posterior density, comparison against the prior mean, which is the fitted value generated from the autoregressive model ignoring the survey data, tells us about the value of the qualitative survey data.

While the RMSE of the indicator $D_{i, t-k}$ is, in general, lower than the benchmark, purely autoregressive indicator, the differences in Table 7 are extremely small. In addition, the improvement in correlation, in all cases but one, is also very minimal. This indicates that while, as seen in Tables 5 and 6, the ITS data do contain a signal about the MPI data this signal does not translate into noticeably improved predictive power for the MPI relative to benchmark autoregressive forecasts. Consistent with Figure 2, where it was seen that $\Delta x_{i, t}$ does not help explain firms' contemporaneous qualitative responses, Table 7 shows that what gains there are to using the posterior indicator, which as indicated are minimal, are confined to lags of $\Delta x_{i, t}$. Therefore, in the case of most interest, we find that conditioning our forecast of the latest MPI data, $\Delta x_{i, t}$, on the latest ITS data, which are available ahead of the MPI data, does not deliver more accurate nowcasts.

Table 8 shows that a similar picture again emerges at the macroeconomic level: conditioning autoregressive (in-sample) forecasts of $\Delta x_{t-k}$ on contemporaneous and lagged values of the balance statistic appears to deliver, at best, minimal gains, with the greater gains again confined to lags of $\Delta x_{t}$. The posterior mean nowcasts in Table 8 are based on estimation of an $\operatorname{ARDL}(11-k, q)$ model; this involved, as with the widely used quantification approach of Pesaran $(1984,1987)$ (e.g., see Driver \& Urga (2004)), regressing 
Table 7: Predictive performance of the ITS indicator of firm-level MPI turnover growth (summarised by its correlation and Root Mean Squared Error)

\begin{tabular}{l|cccc}
\hline & \multicolumn{2}{|c}{$D_{i, t}$ : Posterior Mean } & \multicolumn{2}{c}{ Prior Mean } \\
& $\mathrm{AR}(11)$ & $\mathrm{AR}(1)$ & $\mathrm{AR}(11)$ & $\mathrm{AR}(1)$ \\
\hline Corr with $\Delta x_{i, t}$ & 0.565 & 0.402 & 0.564 & 0.401 \\
RMSE & 0.205 & 0.228 & 0.205 & 0.228 \\
\hline & \multicolumn{2}{|c}{$D_{i, t-1}:$ Posterior Mean } & \multicolumn{2}{c}{ Prior Mean } \\
& $\mathrm{AR}(10)$ & $\mathrm{AR}(1)$ & $\mathrm{AR}(10)$ & $\mathrm{AR}(1)$ \\
\hline Corr with $\Delta x_{i, t-1}$ & 0.554 & 0.417 & 0.543 & 0.402 \\
RMSE & 0.212 & 0.231 & 0.214 & 0.233 \\
\hline & $D_{i, t-2}:$ Posterior Mean & Prior Mean \\
& $\mathrm{AR}(9)$ & $\mathrm{AR}(1)$ & $\mathrm{AR}(9)$ & $\mathrm{AR}(1)$ \\
\hline Corr with $\Delta x_{i, t-2}$ & 0.515 & -0.129 & 0.512 & -0.395 \\
RMSE & 0.215 & 0.252 & 0.215 & 0.253 \\
\hline
\end{tabular}

$D_{i, t}$ is the mean of the posterior density. The prior mean is from the autoregressive model, which ignores the survey data.

the official data (manufacturing output growth $\left.\Delta x_{t}\right)$ on $p=k+1, \ldots, 11$ lags $\left(\Delta x_{t-p}\right)$ and current and $(q-1)$ lagged values of the balance statistic from the ITS. The fact that the conclusions from the firm-level and macro indicators are very similar suggests that the firm-level results are not distorted by the fact that the MPI examines sales while the ITS asks about output volumes. The absolute values of the RMSEs are much lower with the aggregate data than with the firm data for the simple reason that aggregate output is much more stable than is firm-level turnover.

Given the firm-level results in Table 7 it should not perhaps be a surprise to find that it is hard at the macroeconomic level, systematically over time, to beat autoregressive (benchmark) forecasts. But a more general point to bear in mind is that autoregressive models tend to perform very well in periods of stability, while they can often be outclassed in more volatile periods. These results show that, in the stable period examined, early estimates of output growth generated using the CBI survey are very little different from those which can be computed without using the qualitative survey. 
Table 8: Predictive performance of the ITS balance statistic for aggregate manufacturing output growth relative to a benchmark (prior) AR model in manufacturing output growth (summarised by its correlation and Root Mean Squared Error)

\begin{tabular}{c|cccc}
\hline & \multicolumn{2}{|c}{ Posterior Mean of $\Delta x_{t}$} & \multicolumn{2}{c}{ Prior Mean } \\
& ARDL $(11,3)$ & ARDL $(1,3)$ & $\operatorname{AR}(11)$ & $\operatorname{AR}(1)$ \\
\hline Corr with $\Delta x_{t}$ & 0.403 & 0.345 & 0.397 & 0.302 \\
RMSE $\times 100$ & 0.855 & 0.877 & 0.857 & 0.890 \\
\hline & \multicolumn{2}{|c}{ Posterior Mean of $\Delta x_{t-1}$} & Prior Mean \\
& ARDL $(10,3)$ & ARDL $(1,3)$ & AR $(10)$ & AR $(1)$ \\
\hline Corr with $\Delta x_{t-1}$ & 0.427 & 0.371 & 0.399 & 0.305 \\
RMSE $\times 100$ & 0.845 & 0.868 & 0.857 & 0.891 \\
\hline & \multicolumn{2}{|c}{ Posterior Mean of $\Delta x_{t-2}$} & Prior & Mean \\
& ARDL $(9,3)$ & ARDL $(1,3)$ & AR $(9)$ & AR $(1)$ \\
\hline Corr with $\Delta x_{t-2}$ & 0.435 & 0.383 & 0.399 & 0.308 \\
RMSE $\times 100$ & 0.843 & 0.865 & 0.858 & 0.891 \\
\hline
\end{tabular}

\section{Conclusion}

This paper first tests the reliability of qualitative business survey data against official quantitative data at the firm level, as well as, which is more common, at the macroeconomic level. The firm level exercise involved construction of a unique dataset, which involved matching a panel of firms' responses to the qualitative and quantitative surveys. This new dataset is then analysed to provide a definitive means of assessing the informational content of qualitative business surveys of the type routinely analysed by economists. These types of surveys have the perceived advantage of timeliness relative to official surveys. Moreover, they ask firms a range of questions not posed in official surveys. But, as this paper explains, for those questions, like the retrospective output question, which have a natural counterpart in official surveys, it is possible to test the informational content of the qualitative data against the quantitative data at the firm-level. Clearly the approach we suggest could, with the cooperation of data producers, be applied to related surveys in other countries.

Our application to firm-level data from the ITS finds that the retrospective qualitative data are plainly related to the responses the same firms gave to the MPI. Firms also appear to follow the CBI's instructions that they should report on output movements over the last three or before July 2003, four months, 'quite' closely with the peak signal two relative to three months ago. However, on balance, the statistical evidence points to the signal remaining statistically significant up to about six months in the past; although 
it is explained that, given the MPI asks about sales or turnover while the ITS asks about output, this can arise even when firms do follow the CBI's instructions if unexpected fluctuations in sales growth are met from stocks. A clearer conclusion is that the firmlevel qualitative data do not provide a good coincident indicator of growth. This was confirmed when, having introduced a novel means of inferring the official quantitative data from the qualitative data, we found that conditioning autoregressive forecasts of the MPI data on contemporaneous values of the ITS data does not improve inference more than trivially. This suggests that the CBI survey has little role to play in enhancing our knowledge of what has recently happened to manufacturing output, a result which is confirmed when we examine the signal generated by macro-economic data.

Nevertheless, the finding that the responses to the ITS are related to the quantitative returns provided by the same firms in the MPI does suggest that confidence can be placed in the responses to questions which have no counterpart in official enquiries. In the monthly survey these include questions about order books for domestic and export sales and levels of stocks. In the wider quarterly survey there are also questions covering, among other things, business optimism, capital expenditure plans, capacity utilisation, factors likely to limit output and expenditure on training. While verification of these is obviously desirable, the results we find in the first part of our study provide no reason to doubt that the ITS offers valid indicators of the business environment. 


\section{A Appendix}

\section{A.1 The MPI}

There are about 160,000 businesses in the sector covered by the MPI and the overall sample size in each month is about 9000, of which 600 receive employment only forms. The sampling unit of the MPI survey is the reporting unit. The reporting unit of a firm holds the mailing address to which the form is sent. The form can thus cover the whole enterprise, or parts of the enterprise identified by lists of local units. The response rate to the MPI is quite high, reaching over $80 \%$ at the time of publication. This corresponds to around 8000 responses each month.

The MPI adopts stratified random sampling, stratifying the population by industry and employment. 201 industries (in the production sector of SIC2003) are asked to provide turnover and employment data, except for the water supply industry. In addition, 19 industries are surveyed each month for employee counts only. Inputs to the IoP for the remaining production industries not covered by the MPI turnover survey are supplied by trade associations and other government departments.

Stratification takes place by number of employees, with strata: 0-9 employees, 1049 employees, 50-149 employees and 150 or more employees. For most industries the sampling fractions of these strata are 1\%,6\%,16\% and 100\% respectively. However, for some industries where there are not very many firms in the third stratum, all firms employing 50 people or more are included. In total about $63 \%$ of economic activity, measured by employment, is included in the sample.

Samples for the MPI are derived from the IDBR via a Permanent Random Number system (Ohlsson 1995), which allows gradual rotation of the sample within each stratum for each four-digit industry. Sampled firms stay in the sample for a period of months and then receive a "holiday period" which allows them to stay out of the sample. However, those firms in the fully enumerated sizeband stay in the sample permanently. Full details are available in ONS (2005).

The MPI measures sales rather than output. To derive the latter it is necessary to account for any increase in the volume of stocks held. This is measured by a quarterly survey, the Quarterly Inventories Inquiry. Monthly figures are interpolated by splining and the monthly IoP is computed using an extrapolation of these. 


\section{A.2 The ITS}

The ITS is carried out using a full quarterly survey and a more limited survey which includes only five questions selected from the quarterly survey form in the intervening months. The participating UK manufacturing firms are required to give qualitative information about their past and future expectations on their volume of output, costs, prices, business confidence, employment, and some other related questions. Firms are removed from the sample i) if they go out of business or ii) they decide not to participate further. The sample size has gradually decreased over time. The majority of the ITS survey forms are sent to the headquarters or parent companies who generally respond on behalf of their UK-based activities. In the cases of small or medium enterprises, forms are sent to their sole addresses. There were over our sample period, see Table 1, around 800 respondents to the ITS survey each month.

\section{A.3 A two-equation model for output growth and sales growth}

The general model, (7), as long as a sufficient number of lagged terms in $\Delta x_{i, t}$ are included, can be motivated as the solution of a two-equation model, which accommodates the potential endogeneity of sales growth. For a related model see Smith \& Blundell (1986); see also the discussion in Pesaran (1997) which shows, in the context of the ARDL models introduced above for the macroeconomic data, the importance of augmenting the lag order to accommodate the potential endogeneity of the explanatory variables. In our context, endogeneity could arise from the measurement error in relating output growth to sales growth. Let $s_{i, t}^{*}$ represent sales growth, where $s_{i, t}^{*}$ is a linear function of $\Delta x_{i, t}$ and up to $l^{*}<11$ lags, such that $s_{i, t}^{*}=f\left(\Delta x_{i, t}, \Delta x_{i, t-1}, \ldots, \Delta x_{i, t-l^{*}}\right)$. Then consider the two-equation system:

$$
\begin{aligned}
& y_{i, t}^{*}=\varkappa_{i} s_{i, t}^{*}+\alpha_{i}+u_{i, t}^{1} \\
& s_{i, t}^{*}=\pi_{i} s_{i, t-1}^{*}+u_{i, t}^{2}
\end{aligned}
$$

where $\left|\pi_{i}\right|<1$ to ensure stationarity of sales growth, and in the first equation, A-1, output growth, $y_{i, t}^{*}$, relates to sales growth, $s_{i, t}^{*}$, plus an error term, $u_{i, t}^{1}$, which might in part capture the change in stock movements. For notational ease only we suppress dependence of $y_{i, t}^{*}$ on the lagged dummies, $y_{i, t}^{(j)}$. The second equation, $(\mathrm{A}-2)$, then assumes that sales growth follows an autoregressive process, that is taken to be first order. Additional lagged terms can be included if necessary. $\left(u_{i, t}^{1}, u_{i, t}^{2}\right)$ are assumed to be mean zero and 
jointly normally distributed random variables with variances $\sigma_{11}$ and $\sigma_{22}$, respectively, and covariance $\sigma_{12}$. Under these assumptions $u_{i, t}^{1}=\left(\sigma_{12} / \sigma_{22}\right) u_{i, t}^{2}+\epsilon_{i, t}$, where $\epsilon_{i, t}$ is a mean zero disturbance distributed independently of $u_{i, t}^{2}$. When $\sigma_{12}=0$ fluctuations in sales growth are not met from stocks and lead directly to output movements. When $\sigma_{12} \neq 0$, $s_{i, t}^{*}$ is endogenous (correlated with $u_{i, t}^{1}$ ) and the derivation of the relationship between $y_{i, t}^{*}$ and $s_{i, t}^{*}$ should allow for this contemporaneous feedback (or indirect relationship). This is achieved by substituting in (A-1) which is then seen, by augmenting the lag order of (A-1), to generate a (conditional) model of the type seen in (7). The coefficients on $\Delta x_{i, t}$, and its lags, in (7) can then be seen to be a non-linear function of $\varkappa_{i},\left(\sigma_{12} / \sigma_{22}\right), \pi_{i}$ and the assumed functional form for $f\left(\Delta x_{i, t}, \Delta x_{i, t-1}, \ldots, \Delta x_{i, t-l^{*}}\right)$. So while we might expect $\sigma_{12}$ to be negative, if unexpected fluctuations in sales growth (shocks to $u_{i, t}^{2}$ ) are met from stocks, rather than increases or decreases in output, we do not have a prior view on the sign of the coefficients on $\Delta x_{i, t}$, and its lags, in (7). 


\section{References}

Ashley, J., Driver, R., Hayes, S. \& Jeffery, C. (2005), 'Dealing with data uncertainty', Bank of England Quarterly Bulletin Spring, 23-29.

Butler, J. \& Moffit, R. (1982), 'A computationally efficient quadrature procedure for the one-factor multinomial probit model', Econometrica 50, 761-764.

Clements, M. P. \& Hendry, D. F. (1998), Forecasting Economic Time Series, Cambridge University Press: Cambridge.

Deely, J. L. \& Lindley, D. V. (1981), 'Bayes empirical Bayes', Journal of the American Statistical Association 76, 833-841.

Dixon, W. (1960), 'Simplified estimation from censored normal samples', Annals of Mathematical Statistics 31, 385-391.

Driver, C. \& Urga, G. (2004), 'Transforming qualitative survey data: performance comparisons for the UK', Oxford Bulletin of Economics and Statistics 66, 71-90.

Gourieroux, C., Monfort, A., Renault, E. \& Trognon, A. (1987), 'Generalized residuals', Journal of Econometrics 34, 5-32.

Hendry, D. F., Pagan, A. R. \& Sargan, J. (1984), Dynamic specification, in Z. Griliches \& M. D. Intriligator, eds, 'Handbook of Econometrics'.

Hsiao, C., Pesaran, M. H. \& Pick, A. (2007), Diagnostic tests of cross section independence for nonlinear panel data models. Cambridge Working Papers in Economics No.0716.

Inoue, A. \& Kilian, L. (2004), 'In-sample or out-of-sample tests of predictability: Which one should we use?', Econometric Reviews 23, 371-402.

Lui, S., Mitchell, J. \& Weale, M. R. (2009), Collective sentiment in qualitative business survey. National Institute of Economic and Social Research Discussion Paper No.328.

Machin, S. J. \& Stewart, M. B. (1990), 'Unions and the financial performance of British private sector establishments', Journal of Applied Econometrics 5, 327-350.

Mitchell, J., Smith, R. J. \& Weale, M. R. (2002), 'Quantification of qualitative firm-level survey data', Economic Journal 112, C117-C135. 
Mitchell, J., Smith, R. J. \& Weale, M. R. (2005), 'Forecasting manufacturing output growth using firm-level survey data', The Manchester School 73, 479-499.

Mitchell, J., Smith, R. J. \& Weale, M. R. (2006), 'A bayesian indicator of manufacturing output from qualitative business panel survey data'. National Institute of Economic and Social Research Discussion Paper No.261 (Revised)).

Ohlsson, E. (1995), Co-ordination of samples using permanent random numbers, in B. Cox, D. Binder, B. Chinnapa, A. Christianson, M. Colledge \& P. Kott, eds, 'Business Survey Methods', Wiley, New York, pp. 153-169.

Olsson, U., Drasgow, F. \& Dorans, N. J. (1982), 'The polyserial correlation coefficient', Psychometrika 47, 337-347.

ONS (2005), Report on the full triennial review of the monthly production inquiry. Available at http://www.statistics.gov.uk/downloads/reviews/MPItriennialReport2005.pdf.

ONS (2007), General information on the Quarterly Inventory (Stocks) Inquiry, note $=$ Available at http://www.statistics.gov.uk/StatBase/Source.asp?vlnk=1444\&More=Y.

ONS (2009), A Brief Guide to the IDBR. http://www.statistics.gov.uk/idbr/idbr.asp.

Pesaran, M. H. (1984), Expectations formation and macroeconomic modelling, in P. Malgrange \& P. Muet, eds, 'Contemporary Macroeconomic Modelling', Blackwell, Oxford, pp. 27-55.

Pesaran, M. H. (1987), The limits to rational expectations, Basil Blackwel, Oxford.

Pesaran, M. H. (1997), 'The role of economic theory in modelling the long run', Economic Journal 107(440), 178-91.

Pesaran, M. H. (2006), 'Estimation and inference in large heterogeneous panels with a multifactor error structure', Econometrica 74(4), 967-1012.

Pesaran, M. H. \& Weale, M. R. (2006), Survey Expectations, in G. Elliott, C. W. J. Granger \& A. Timmermann, eds, 'Handbook of Economic Forecasting Volume 1', North-Holland, pp. 715-776. 
Smith, R. J. \& Blundell, R. W. (1986), 'An exogeneity test for a simultaneous equation Tobit model with an application to labor supply', Econometrica 54, 679-685.

Verbeek, M. \& Nijman, T. (1992), 'Testing for selectivity bias in panel data models', International Economic Review 33, 681-703. 